\title{
Single-Strand DNA Breaks Cause Replisome Disassembly
}

${ }^{1}$ Department of Biological Chemistry and Molecular Pharmacology, 


\section{Summary}

22 DNA damage impedes replication fork progression and threatens genome stability. Upon

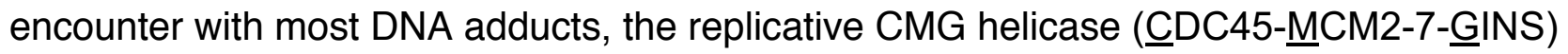

24 stalls or uncouples from the point of synthesis, yet CMG eventually resumes replication.

25 However, little is known about the effect on replication of single-strand breaks or "nicks", which are abundant in mammalian cells. Using Xenopus egg extracts, we reveal that CMG collision with a nick in the leading strand template generates a blunt-ended double-strand break (DSB). Moreover, CMG, which encircles the leading strand template, "runs off" the end of the DSB. In contrast, CMG collision with a lagging strand nick generates a broken end with a single-stranded overhang. In this setting, CMG translocates beyond the nick on doublestranded DNA and is then actively removed from chromatin by the p97 ATPase. Our results show that nicks are uniquely dangerous DNA lesions that invariably cause replisome disassembly, and they argue that CMG cannot be deposited on dsDNA while cells resolve replication stress.

\section{Keywords}

DNA replication, DNA repair, single molecule, fork collapse, replisome, helicase, doublestrand break, single-strand break, CMG, homologous recombination

\section{Highlights}

- The structures of leading and lagging strand collapsed forks are different

- CMG passively "runs off" the broken DNA end during leading strand fork collapse

- $\quad$ CMG is unloaded from duplex DNA after lag collapse in a p97-dependent manner

- Nicks are uniquely toxic lesions that cause fork collapse and replisome disassembly 
bioRxiv preprint doi: https://doi.org/10.1101/2020.08.17.254235; this version posted August 17, 2020. The copyright holder for this preprint (which was not certified by peer review) is the author/funder. All rights reserved. No reuse allowed without permission. 


\section{Introduction}

Single-stranded DNA breaks or "nicks" are generated by ionizing radiation, free radicals, topoisomerase I, and as intermediates in base excision repair (BER) (Caldecott, 2008). Nicks encountered in the leading strand template during eukaryotic replication cause replication fork "collapse" with formation of a single-ended double-strand break (seDSB; Figure S1A) (Nielsen et al., 2009; Strumberg et al., 2000), but whether this also occurs at lagging strand nicks has not been examined. seDSBs can be repaired by a subpathway of homologous recombination (HR) known as break-induced replication (BIR), which involves resection of the broken end, invasion into the sister chromatid, and replication to the end of the chromosome or until a converging fork is encountered (Haber, 1999; Mayle et al., 2015). Loss of the recombination proteins $\mathrm{RAD51}$, BRCA1, or BRCA2 is lethal in unperturbed vertebrate cells (Hakem et al., 1996; Sharan et al., 1997; Sonoda et al., 1998; Tsuzuki et al., 1996), consistent with estimates that $\sim 50$ replication forks normally collapse in every $\mathrm{S}$ phase (Vilenchik and Knudson, 2003). Moreover, cancer therapeutics such as topoisomerase and poly (ADP-ribose) polymerase (PARP) inhibitors function by stabilizing nicks and promoting replication fork collapse (Hengel et al., 2017). Thus, the repair of collapsed forks is essential for cell viability and represents a prominent target in cancer therapy.

A central question is whether the replicative helicase CMG participates in replication restart after fork collapse. Early work suggested that CMG participates in BIR and that it remains on chromatin during fork collapse (Hashimoto et al., 2012; Lydeard et al., 2010). Moreover, a recent single molecule study suggested that when replication forks encounter DNA damage, CMG moves onto parental DNA beyond the damage, and when repair is complete, it re-engages with the fork for replication restart (Wasserman et al., 2019). However, other studies conclude that CMG is not involved in BIR (Dilley et al., 2016; Natsume et al., 2017; Sonneville et al., 2019; Wilson et al., 2013). To determine whether 
71 CMG could function in replication restart, tracking its fate during replication stress in a

72 physiological setting is crucial. Another key factor dictating repair is the DNA structure

73 generated during fork collapse. The specific structure formed may depend on whether

74 collapse occurs at a nick in the leading strand template (Figure S1A,i, hereafter "lead

75 collapse") or lagging strand template (Figure S1A,ii, hereafter "lag collapse"). Work in human

76 cells suggests that during lead collapse, a blunt, seDSB is generated (Strumberg et al., 2000)

77 (Figure S1A,i) that would have to undergo resection prior to strand invasion. However, the lag

78 collapse fork structure is unknown. In summary, whether the replisome is recycled for BIR

79 and which DNA structures and processing steps underlie this clinically relevant DNA repair

80 pathway remain unclear.

Here, we use ensemble and single molecule approaches in Xenopus egg extracts to

examine the fate of the replication fork after collision with strand-specific nicks. Strikingly,

83 after both lead and lag collapse, the CMG helicase is lost from the DNA, but via distinct

84 mechanisms. Further, analysis of the fork DNA structures generated shows that lag collapse

85 is more amenable to repair than lead collapse. Our results establish replication fork collision

86 with nicks as uniquely deleterious for replication fork progression but also indicate that there

87 are important differences between lead and lag collapse.

89 Results

Lead collapse generates a nearly blunt seDSB and a gap in the lagging strand

91 We wanted to study DNA replication fork collapse at a site-specific nick using Xenopus egg

92 extracts, which support sequence non-specific replication initiation on added DNA, followed

93 by a complete round of replication (Figure S1B) (Walter et al., 1998). However, nicked

94 plasmid added to egg extract was rapidly ligated (Figure S1C, lanes 1-6) before forks would 
95

96

97

98

be able to reach the nick. Chemical modification of nucleotides flanking the nick did not prevent ligation (data not shown). We therefore flanked the nick with Tet operator (tetO) sites to which we bound the Tet repressor (TetR) before adding the plasmid to extract. We reasoned that TetR might stabilize the nick by blocking access to DNA ligase. Indeed, although TetR did not block fork progression (see Figure 3D legend below), it increased the half-life of the nick $\sim 45$-fold (Figure S1C). To further increase the probability that forks encounter a nick, we used three consecutive nicks, each flanked by tetO sites (Figure 1A, inset). To compare fork collapse when nicks reside in the leading versus lagging strand templates, it was necessary to ensure that forks arrive at the nicks from only one direction. Therefore, we flanked the nicks on the right with an array of 48 Lac repressors (LacR) (Figure 1A), which blocks replication fork progression in egg extracts (Dewar et al., 2015; Duxin et al., 2014), preventing arrival of a second fork at the nick. In this configuration, lead collapse should occur when the rightward fork collides with a nick in the bottom strand (Figure 1A, inset).

When we replicated unnicked plasmid in extracts containing LacR and TetR, a prominent "theta" intermediate was generated (Figure S1D, lanes 1-3), as expected from forks stalling at the outer edges of the LacR array (Duxin et al., 2014). However, in the presence of a nick, a prominent new product appeared that migrated faster than theta (Figure S1D, lanes 4-6, red arrowhead). To identify the structure of this new product, we cut out the band, extracted the DNA, and performed transmission electron microscopy. This analysis revealed that the new product corresponds to a sigma-shaped, "collapsed" structure in which a linear arm of the expected size (2.7 kb; Figures $1 \mathrm{~A}$ and $\mathrm{S} 1 \mathrm{E})$ is attached to the circular plasmid (Figure 1B, red arrow). Quantification showed that $\sim 60 \%$ of the forks from the nicked 
118 plasmid underwent collapse (Figure S1D, lane 4). We attribute the remaining theta products

119 (Figures 1A and 1B, bottom image; Figure S1D) to plasmids in which all three nicks were

120 ligated before fork arrival. Our data provide direct visual confirmation of previous reports

121 (Nielsen et al., 2009; Strumberg et al., 2000) that replication through a leading strand nick

122 causes fork collapse with formation of a seDSB.

To determine the DNA structure of the broken end generated during lead collapse, we

124 labeled nascent DNA stands with $\left[\alpha-{ }^{32} \mathrm{P}\right] \mathrm{dATP}$ and used the single strand endonuclease

125 Nt.BspQI to cleave these strands at a defined position relative to the three TetR-flanked nicks

126 (Figure 1C). Denaturing gel analysis showed that replication through the nick generated three

127 ssDNA products whose 3' ends were located close to each of the three nicks (Figure 1D,

128 lanes 5-8). The product associated with collapse at the first nick was most abundant (Figure

$1291 \mathrm{E})$, as expected from rightward fork movement. Higher resolution mapping showed that

130 leading strand synthesis mostly stopped $3 \mathrm{nt}$ from the end of the break, which should leave a

$1313 \mathrm{nt}, 5^{\prime}$ 'single-stranded DNA overhang (Figures S1F and S1G, lanes 5-8). This interpretation

132 is consistent with the properties of purified pol $\varepsilon$, the leading strand polymerase (Hogg et al.,

133 2014). Our results suggest that lead collapse repair should generally require resection of the

134 seDSB.

To map the distribution of nascent lagging strands during lead collapse, we next

136 placed the Nt.BspQI site on the other strand (Figure 1F). Most nascent lagging strands were

$137440-460$ nts in length (Figure 1G; purple bar). Because the first nick is located 518 basepairs

138 from the Nt.BspQI site, we conclude that there is typically a 60-80 nt gap between 5' end of

139 the nascent lagging strand and the site of collapse (Figure 1F, "gap"). By 20 minutes, lagging

140 strand fragments declined, indicating gap filling (Figure 1G, lanes 3-4). Consistent with this 
141 interpretation, well-defined bands appeared that correspond to gap-filled products that retain

142 the second and third nicks due to TetR (Figures 1F and 1G,i,ii). In summary, lead collapse

143 generates a seDSB with a three nucleotide 5' overhang and a lagging strand gap that is

144 subsequently filled in. The data suggest that lead collapse repair involves not only resection

145 but also gap filling.

147 CMG is lost at the nick after lead collapse

148 Given that the leading strand is extended to within 3 nucleotides of the break, and because

149 the CMG footprint on DNA comprises 20-40 nt (Fu et al., 2011), our data suggest that CMG

150 loses its association with the DNA end during lead collapse. However, whether CMG

151 dissociates altogether was unclear. To address this question, we used single molecule

152 imaging to visualize CMG dynamics during lead collapse. We replicated stretched,

153 immobilized DNA in GINS-depleted extract containing Alexa Fluor 647-labeled recombinant

154 GINS (GINS ${ }^{A F 647}$ ), a subunit of CMG (Figure S2A). Extracts also contained fluorescent Fen1

155 (Fen1 ${ }^{\mathrm{mKikGR}}$ ) to image nascent DNA synthesis (Loveland et al., 2012). As reported previously

156 (Sparks et al., 2019), total internal reflection fluorescence microscopy revealed GINS ${ }^{\mathrm{AF} 647}$

157 molecules moving at the leading edge of growing Fen1 ${ }^{\text {mKikGR }}$ tracts, demonstrating that

158 GINS ${ }^{\mathrm{AF} 647}$ travels with active replication forks (Figures 2A,i, Supplemental Video 1). To

159 generate a fluorescently labeled nick, we used a point mutant of Cas9, H840A ("nCas9"),

160 which selectively nicks the non-target DNA strand (Jinek et al., 2012). nCas9 promoted

161 efficient replication fork collapse (compare Figure S2B to Figure S1D). Furthermore, nCas9

162 RNPs labeled at the $5^{\prime}$ end of the tracrRNA with Atto550 (nCas9Atto550; Figure 2B) bound

163 specifically to the target site on the stretched DNA (Figure S2C). However, nCas9Atto550

164 dissociated at a significant rate upon exposure to extract (Figures S3D and S3E; (Wang et 
165 al., 2020)). Therefore, to increase the likelihood of fork collapse, nCas9Atto550 was targeted to 4 sites in the same strand located $\sim 1 \mathrm{~kb}$ apart. In most cases, only 1-2 nCas 9 molecules

167 remained bound at the time of fork arrival. The cluster of nicks was positioned $5-8 \mathrm{~kb}$ from

168 one end of the $30 \mathrm{~kb}$ DNA such that a fork traveling along the short arm underwent lead

169 collapse when it collided with nCas9 (Figure 2A,ii, Supplemental Video 2). Representative examples of lead collapse are shown in Figure 2A,ii. In this scenario,

171 CMG collided with nCas9, paused for a few minutes, and then disappeared. In $85 \%$ of such

172 collisions, GINS dissociated from the chromatin at the nick site (Figure S2Fi,ii,vi). In contrast, when a catalytically dead mutant of Cas9 (dCas9) was targeted to these sites, CMG typically paused at dCas9 and then resumed replication (Figure 2A,iii, Figure S2G,iii, and

177 lost at the same time as nCas9Atto550 $45 \%$ of the time (Figure S2F,i). Surprisingly, GINS ${ }^{A F 647}$ was lost before nCas9Atto550 $33 \%$ of the time, suggesting that nCas9 may sometimes retain its

179 interaction with DNA even after CMG slides off the end of the break (Figure S2F,ii). Such an 180 outcome is plausible because CMG should be able to slide off the leading strand template without disrupting most of the contacts that nCas9 makes with the lagging strand template 182 (Figure 2B, lead collapse). Instances in which CMG passes the nick site (Figure S2F,v) can mostly be attributed to the absence of a nick, as seen for dCas9, likely because nCas9 had not cleaved the DNA at the time of collision (see below). In summary, the loss of GINSAF647 at

185 the nick site concurrent with or before nCas9 dissociation supports the model that CMG fully dissociates from the end of the break during lead collapse. 
What happens to the replication fork during lag collapse is unknown. To address this

question, we replicated a plasmid with TetR-protected nicks on the lagging strand template

191 (top strand) (Figure S3A). As shown in Figure S3B, this yielded the same new band observed

during lead collapse (lanes 7-12). To determine the structure of the seDSB generated during

lag collapse, we cleaved nascent lagging strands 515 bp from the first nick (Figure 3A).

Denaturing gel electrophoresis showed that their 5' ends were typically located 60-80

nucleotides from the break point (Figure 3B, purple bar), consistent with random initiation of

the last Okazaki fragment relative to the nick. Therefore, lag collapse results in a seDSB

break with a $70 \mathrm{nt} 3^{\prime}$ ssDNA overhang. Analysis of the nascent leading strands revealed that

they were extended to within a few nucleotides of each nick (Figures 3C and 3D), and some

were extended a few nucleotides beyond the nick, consistent with limited strand displacement

argues that CMG does not stall when it hits the nick (Figure $3 \mathrm{C}, \mathrm{i}$ ) because no stalling

products were detected 20-40 nt upstream of the nick (Figure 3D). Moreover, leading strand

arrest at the nick also rules out that CMG continues unwinding DNA well beyond the nick

(Figure 3C,ii). Instead, the data suggest that CMG either rapidly dissociates at the nick

(Figure 3C,iii), or that it somehow translocates beyond the nick without unwinding DNA

(Figure 3C,iv), either of which would allow leading strand synthesis to reach the nick.

\section{Active CMG unloading from double-stranded DNA during lag collapse}

209 To distinguish between the latter two models, we examined the fate of fluorescent CMGs

210 during lag collapse, which occurs when CMGs collide with nCas9Atto550 from the long arm of

211 immobilized DNAs (Figure 4A, Supplemental Video 4). As seen for lead collapse (Figure

212 2A,ii), CMG typically paused at the nCas9, followed by rapid loss of nCas9 and CMG (Figure 
$2134 \mathrm{~A}$, Veh.). However, far fewer CMGs were lost at the same time as nCas9 during lag collapse

214 vs. lead collapse (4\% vs. 45\%; Figures. S2H,i and S2F,i), and more CMGs persisted longer

215 than nCas9 for lag collapse compared to lead collapse (59\% vs. 21\%; Figures S2H,iii and

$216 \mathrm{~S} 2 \mathrm{~F}$,iii). Together, our results demonstrate that CMG is lost from chromatin during lead and

217 lag collapse, but the delayed CMG unloading during lag collapse could suggest a mechanism

218 involving multiple steps.

We hypothesized that CMG unloading during lag collapse might involve the same

220 mechanism used during replication termination, in which converging CMGs are ubiquitylated

221 by CRL2 ${ }^{\mathrm{Lrr}}$ and unloaded by the p97 ATPase (Figure S4A) (Dewar and Walter, 2017). To

222 test this idea, we supplemented extracts with MLN4924 (“Cul-i”), which inhibits all cullin-RING

223 E3 ubiquitin ligases including CRL2 ${ }^{\mathrm{Lrr1}}$ (Dewar et al., 2017), or NMS873 ("p97-i”), an allosteric

224 p97 inhibitor. Strikingly, Cul-i delayed CMG dissociation 9-fold after collision with lagging

225 strand nicks, and the retained CMG continued unidirectionally translocating along the DNA

226 away from the collapse site (Figures 4A and 4B, Supplemental Video 5). Similarly, p97-i

227 inhibited CMG unloading and promoted continued translocation beyond the nick (Figures 4A

228 and 4B, Supplemental Video 6). Importantly, Cul-i and p97-i did not affect CMG loss during

229 lead collapse (Figure 4B). These results show that CMG unloading during lag collapse, but

230 not lead collapse, involves a cullin-RING ligase and the p97 ATPase, as seen during

231 termination.

232 CMG translocates onto dsDNA before being unloaded during termination (Dewar et

233 al., 2015)(Low et al., in preparation). Our data suggest that a similar phenomenon occurs

234 during lag collapse. When CMG translocated beyond a lagging strand nick in the presence of

235 Cul-i or p97-i, no Fen1 signal was detected behind the helicase $85 \%$ and $84 \%$ of the time, 
respectively (Figure S2H,ix). Similarly, no Fen1 signal was observed when CMG translocated beyond the nick in the absence of Cul-i and p97-i (Figures S4C and S2H,ix), but such events were rare because CMG was usually unloaded before it could travel far enough to assess Fen1's behavior (Figures S4D and S2H,viii). Thus, CMG translocation beyond the nick after

240 lag collapse is not associated with DNA synthesis. Moreover, after colliding with a lagging

241 strand nick, CMG translocated three-fold slower than before the collision (Figure 4C; 144

$242 \mathrm{bp} / \mathrm{min}$ vs. $422 \mathrm{bp} / \mathrm{min})$. However, the rate of CMG progression was the same before and 243 after encounter with nCas9 in the few instances in which Fen1 signal did trail behind CMG 244 after the encounter (Figures 4C and S4E). Thus, we infer that the fork failed to undergo 245 collapse in these cases (Figures S2F,ix and S2H,x). In summary, after CMG travels beyond a 246 lagging strand nick, it translocates slowly and does not promote DNA synthesis. This strongly 247 implies that CMG transitions onto dsDNA at the nick, which is consistent with biochemical 248 experiments using purified CMG (Kang et al., 2012; Langston and O’Donnell, 2017).

249 Together, the data argue that during lag collapse, CMG is removed from dsDNA via the same 250 mechanism that operates during replication termination (Figure S4A and S4B).

\section{Discussion}

253 In this study, we have monitored the fate of the replisome at nicks, an abundant form of DNA 254 damage. We find that when forks encounter nicks in the leading strand template, CMG undergoes passive dissociation when it slides off the end of the break; at lagging strand 256 nicks, CMG transitions onto dsDNA and is recognized as a terminated complex, leading to 257 active, p97-dependent removal. CMG anchors most proteins to the fork, suggesting that the 
known mechanism to re-assemble CMG de novo in S phase. Therefore, our results strongly suggest that after fork collapse, resumption of DNA synthesis requires nucleation of a BIR

\section{1} replisome around a new DNA helicase. Our results are consistent with studies that BIR involves Pif1 in yeast and possibly MCM8-9 in mammalian cells (Natsume et al., 2017;

Wilson et al., 2013). In summary, our data show that compared to most chemical adducts, replisome disassembly.

The question arises whether CMG can be recycled during other forms of replication replication stalls at DNA damage, CMG diffuses onto dsDNA downstream of the damage; when repair is complete, CMG re-engages with the fork for replication restart (Wasserman et

al., 2019). However, our data show that CMG is rapidly unloaded from dsDNA. Moreover, even if unloading could be prevented, CMG would translocate unidirectionally away from the stressed fork. Thus, our data suggest that in a cellular context, depositing CMG on dsDNA cannot be used to help the fork overcome DNA damage or to maintain the replisome during 274 fork reversal. 
282

283

284

285

probably sufficient to promote strand invasion without further resection (Ira and Haber, 2002;

Jakobsen et al., 2019). Our data predict that lead collapse repair requires more processing steps than lag collapse repair and thus might fail in genetic backgrounds that do not support resection (Nacson et al., 2020). In addition, the nearly blunt DNA end generated during lead collapse is an excellent binding site for Ku, suggesting that lead collapse should be more susceptible than lag collapse to NHEJ-dependent chromosomal translocations.

We previously showed that when forks converge during replication termination, CMG is ubiquitylated by CRL2 ${ }^{\text {Lrr1 }}$ and subsequently unloaded by p97. Remarkably, CMG appears to be unloaded by the same mechanism when a single fork encounters a lagging strand nick. This observation argues that CRL2 $2^{\text {Lrr1 }}$-dependent CMG unloading operates in multiple cellular contexts and that termination-induced CMG unloading does not require the convergence of two forks. Instead, we recently proposed that CMG ubiquitylation is normally suppressed during elongation by the excluded DNA strand, which prevents CRL2 ${ }^{\operatorname{Lr} 1}$ binding to the outer face of CMG. In this model, suppression is lost when the excluded strand dissociates from CMG during termination (Low et al., in preparation), or when CMG passes onto dsDNA at a lagging strand nick (Fig 4d). We hypothesize that CMG removal during replication termination and fork collapse serves the same purpose, namely, to prevent the interference of translocating CMGs with transcription and other chromatin-based processes.

\section{Acknowledgements}

We thank D. Pellman, J. Haber, and members of the Walter laboratory for helpful discussions and comments on the manuscript. This work was supported by the Howard Hughes Medical Institute and NIH Grants HL098316 and GM80676. K.B.V. and R.A.W. are also supported by an American Cancer 
305 Society Postdoctoral Fellowships. G.C. was supported by the Jane Coffin Childs memorial fund.

306 J.C.W. is an investigator of the Howard Hughes Medical Institute.

307

\section{Author Contributions}

309 K.B.V. performed all the experiments shown, except for Figure S3b, which was performed by R.A.W.

310 J.M.D. conceived of, developed, and validated the tet-nick system. T.G.W.G. helped validate the tet-

311 nick system. G.C. developed the CMG single molecule imaging approach, and provided labeled

312 GINS, affinity-purified GINS antibody, and Fen1 for the experiments. J.C.W. supervised the work.

313 K.B.V. and J.C.W. wrote the manuscript, with input from the other co-authors.

\section{Declaration of Interests}

J.C.W. is a co-founder of MoMa therapeutics, in which he has a financial interest.

\section{Methods}

\section{Preparation of nicked plasmids}

321 The nicked plasmids used for the ensemble experiments were generated from a standard pBlueScript

322 plasmid (pBS), with the following modifications. The pBS BspQI site was removed by site-directed

323 mutagenesis using a QuikChange kit (Agilent). New BspQI sites were added to the plasmids either

$324245 \mathrm{bp}$ (to visualize the nascent leading strand) or $497 \mathrm{bp}$ (to visualize the nascent lagging strand)

325 away from the intended Tet-nick location using site-directed mutagenesis with the following primer

326 sets: 1) 245 bp, 5'- ATGGTTCACGTAGTGGCTCTTCGCCATCGCCCTGATAGACG-3' and 5'-

327 GCCACTACGTGAACCATCACCCTAATCAAGTTTTTTGGGG-3'; 2) 494 bp, 5'-

328 CCGAAAAGTGCCACGAAGAGCTGACGCGCCCTGTAGCG-3' and 5'-

329 CGTGGCACTTTTCGGGGAAATGTGCGCGGAACCCC-3'. A Bglll site was added to each plasmid 330 where the Tet-nicks would be inserted using 5'-

331 CCATTCGCCATTCAGAGATCTGCTGCGCAACTGTTG-3' and 5'- 
332 CAACAGTTGCGCAGCAGATCTCTGAATGGCGAATGG-3' primers. Next the plasmids were

333 linearized with Bglll and the first Aflll-Nhel-TetR-BbvCl-TetR-Avrll-Ncol site was added using Gibson

334 assembly with the following sequence as a duplex (only one strand shown): 5'-

335 CAATTTCCATTCGCCATTCAGACTTAAGGCTAGCTCTCTATCACTGATAGGGACCTCAGCTCTCTA

336 TCACTGATAGGGACCTAGGCCATGGAGCTGCGCAACTGTTGGGAAGG-3'. Two additional repeats

337 of these sequences were sequentially added by digesting with Avrll and ligating a Nhel-TetR-BbvCl-

338 TetR-Avrll insert into the plasmids. Finally, two 24x LacO arrays were added between the Sacl and

339 Kpn sites as previously described (Duxin et al., 2014).

$340 \quad$ Plasmids were then run on $0.8 \%$ agarose gels and the supercoiled DNA was extracted via

341 electroelution. Purified plasmids were nicked with $\mathrm{Nb} . \mathrm{BbvCl}$ or $\mathrm{Nt}$. BbvCl for leading or lagging strand

342 fork collapse, respectively. The plasmids were then gel purified by electroelution and stored at $-20^{\circ} \mathrm{C}$

343 in $10 \mathrm{mM}$ Tris, pH 7.5. Plasmid pKV44 has an Nt.BspQI site positioned to nick the nascent leading

344 strand 269 nt away from the first Nb.BbvCl nick site. Plasmid pKV45 has an Nt.BspQI site positioned

345 to nick the nascent lagging strand $515 \mathrm{nt}$ away from the first $\mathrm{Nt.BbvCl}$ nick site. pKV44 was used for

346 all ensemble lead collapse experiments except when we wanted to visualize the lagging strand after

347 lead collapse (then we nicked pKV45 with Nb.BspQI). pKV45 was used for all ensemble lag collapse

348 experiments except when we wanted to visualize the leading strand after lag collapse (then we nicked

349 pKV44 with Nt.BspQI). All restriction and nicking enzymes were purchased from New England Biolabs

350 and used according to manufacturer protocols.

352 Preparation of egg extracts

353 The high-speed supernatant (HSS) and nucleoplasmic extracts (NPE) were prepared from Xenopus

354 laevis eggs as described previously (Lebofsky et al., 2009).

356 Protein expression and purification 
357 Purification of LacR (Dewar et al., 2015) and GINS (Sparks et al., 2019) were described previously.

358 TetR has a His-tag and was purified using Ni-NTA resin as follows. The TetR expression plasmid

359 (TetR gene from addgene plasmid 17492 cloned into pET28b) was transformed into BL21 cells in LB

360 supplemented with $50 \mu \mathrm{g} / \mathrm{mL}$ kanamycin. A single colony from this transformation was used to

361 inoculate LB supplemented with $50 \mu \mathrm{g} / \mathrm{mL}$ kanamycin, which was then grown to an OD600 of 0.5 at

$36237^{\circ} \mathrm{C}$. Expression was induced by adding IPTG to $1 \mathrm{mM}$. After $3 \mathrm{~h}$ incubation, the cells were pelleted,

363 and the supernatant was discarded. The cell pellet was resuspended in $2 \mathrm{~mL}$ lysis buffer (20 mM Tris,

$364 \mathrm{pH}$ 8.0, $1 \mathrm{M} \mathrm{NaCl}, 5 \mathrm{mM}$ imidazole, $1 \mathrm{mM} \mathrm{DTT}, 1$ cOmplete Protease Inhibitor Cocktail tablet (Roche),

$3651 \mathrm{mg} / \mathrm{mL}$ lysozyme) and rotated for $1 \mathrm{~h}$ at $4^{\circ} \mathrm{C}$. Lysate was split into two $1.5 \mathrm{~mL}$ tubes and centrifuged

366 in a microcentrifuge at $4^{\circ} \mathrm{C}$ at 13,000 RPM for $30 \mathrm{~min}$. Supernatant was recovered and applied to

367 equilibrated Ni-NTA resin. Samples were spun with Ni-NTA resin for $1 \mathrm{~h}$ at $4^{\circ} \mathrm{C}$. Resin with lysate was

368 added to a disposable column. Resin was washed twice with $4 \mathrm{~mL}$ of wash buffer (20 mM Tris, $\mathrm{pH}$

$3698.0,1 \mathrm{M} \mathrm{NaCl}, 20 \mathrm{mM}$ imidazole, $1 \mathrm{mM} \mathrm{DTT})$. TetR was eluted from column with four, $0.5 \mathrm{~mL}$

370 additions of elution buffer (10 mL, $20 \mathrm{mM}$ Tris, $\mathrm{pH}$ 8.0, $1 \mathrm{M} \mathrm{NaCl}, 1 \mathrm{mM}$ DTT, $0.5 \mathrm{M}$ Imidazole). DTT (5

$371 \mathrm{mM}$ ) was added to samples immediately after eluting. TetR eluates were combined and dialyzed into

$3721 \mathrm{~L}$ of TetR dialysis buffer (81 mM Tris, pH 7.5, $1.62 \mathrm{mM}$ EDTA, $162 \mathrm{mM} \mathrm{NaCl}, 1.62 \mathrm{mM}$ DTT) for $2 \mathrm{~h}$

373 at $4^{\circ} \mathrm{C}$, then dialyzed into $1 \mathrm{~L}$ dialysis buffer overnight. Dialyzed samples were collected and glycerol

374 was added to bring the glycerol to $38 \%$ of total volume. Samples were aliquoted and stored at $-20^{\circ} \mathrm{C}$.

376 Ensemble fork collapse reactions

377 All ensemble replication reactions were carried out as previously described (Lebofsky et al., 2009), 378 with notable changes mentioned below. Briefly, the replication reactions were performed by first pre-

379 binding the plasmid with TetR and LacR, then licensing the DNA in HSS, and finally addition of NPE

380 to initiate replication. One volume of plasmid DNA was pre-incubated with 3 volumes of LacR (23 $\mu \mathrm{M})$

381 and 3 volumes of TetR $(765 \mu \mathrm{M})$ for 20-30 min. After plasmid DNA was pre-bound with LacR and

382 TetR, the DNA was licensed in HSS at a concentration of $6 \mathrm{ng} / \mu \mathrm{L}$. DNA replication was initiated by 
mixing 1 volume of licensing mix with 2 volumes of NPE mix that was diluted up to $50 \%$ with $1 x$ ELB-

replication stop buffer (8mM EDTA, 0.13\% phosphoric acid, 10\% ficoll, 5\% SDS, 0.2\% bromophenol

for $1 \mathrm{~h}$ at $37^{\circ} \mathrm{C}$, and plasmids were run on a $0.8-1 \%$ agarose gel for $\sim 2.5 \mathrm{~h}$. Gels were then visualized by phosphorimaging on a Typhoon FLA 7000 phosphorimager (GE Healthcare).

\section{Electron microscopy imaging of DNA structures}

Ensemble fork collapse reactions were carried out as described above. After $15 \mathrm{~min}, 50 \mu \mathrm{L}$ of reaction mix was stopped by addition to $420 \mu \mathrm{L}$ extraction stop buffer. The stopped reactions were then RNase and proteinase $\mathrm{K}$ treated. DNA was purified by two rounds of phenol-chloroform extraction followed by ethanol precipitation. DNA was resuspended in $20 \mu \mathrm{L}$ replication stop buffer and was run on a $0.8 \%$ agarose gel for $2 \mathrm{~h}$. The gel was then stained with SYBR gold for $1 \mathrm{~h}$ to visualize the DNA, and DNA bands of interest were visualized and excised on a blue light box. DNA was electroeluted from the gel slices using an EluTrap system and concentrated with Amicon concentrators $(0.5 \mathrm{~mL}, 100 \mathrm{kDa}$ MWCO). ammonium acetate. This mixture was incubated for $10 \mathrm{~min}$. To these mixtures, $2 \mu \mathrm{L}$ of $0.2 \mu \mathrm{g} / \mu \mathrm{L}$ cytochrome c solution was added. These solutions were then placed onto Parafilm as drops and allowed to incubate for $15 \mathrm{~min}$. Next, we lightly touched parlodion-coated grids to the surface of the drops, and we dehydrated each grid in 50\%, 75\%, and $95 \%$ ethanol for 15 s each. Each grid was lightly touched to filter paper to dab off excess solution. In order to increase the contrast of the DNA,

407 the grids were rotary shadowed in a Leica ACE600 with a platinum E-beam using the following 
409 the grids to stabilize the parlodion film using the Leica ACE600 carbon E-beam set to the following

410 parameters: $0^{\circ}$ angle, $130 \mathrm{~W}$ power, $5 \times 10^{-6} \mathrm{mbarr}$, and $2 \mathrm{~nm} \mathrm{C}$ deposition. Grids were examined with

411 a FEI T12 transmission electron microscope (TEM) equipped with a Gatan 2k SC200 CCD camera at

$41240 \mathrm{kV}$ or a JEOL 1200EX TEM equipped with a 2k CCD camera (Advanced Microscopy Techniques).

\section{Nascent strand analysis}

415 To visualize the nascent strands on a denaturing gel, ensemble replication reactions were stopped at 416 indicated time points by mixing 4-6 $\mu \mathrm{L}$ of reaction mix with $30 \mu \mathrm{L}$ extraction stop buffer (0.5\% SDS, 25 $417 \mathrm{mM}$ EDTA, $50 \mathrm{mM}$ Tris- $\mathrm{HCl}, \mathrm{pH}$ 8.0) followed by addition of $5 \mu \mathrm{L} 4 \mathrm{mg} / \mathrm{mL}$ RNase A, and incubation at $37^{\circ} \mathrm{C}$ for $45 \mathrm{~min}$. Next, the proteins were digested with $5 \mu \mathrm{L}$ of $20 \mathrm{mg} / \mathrm{mL}$ Proteinase $\mathrm{K}$ for at least 1.5 $\mathrm{h}$ at $37^{\circ} \mathrm{C}$. Samples were then diluted to $145 \mu \mathrm{L}$ with $10 \mathrm{mM}$ Tris, $\mathrm{pH} 7.5$, and DNA was extracted by phenol-chloroform and ethanol precipitation.

Nascent DNA strands were either digested with Nt.BspQI or AfllI to visualize the collapse

422 products, as noted in the figure legends. First, ethanol precipitated DNA was resuspended in $5 \mu \mathrm{L} 10$

$\mathrm{mM}$ Tris, $\mathrm{pH}$ 7.5. Each of the digestion reactions was carried out using New England Biolabs enzymes

424 and buffers at a final volume of $10 \mu \mathrm{L}$. The Nt.BspQI digestions were carried out with 1 Unit of

425 Nt.BspQI in $1 \times$ NEB3.1 Buffer at $50^{\circ} \mathrm{C}$ for $1 \mathrm{~h}$. The Aflll digestions were carried out with 2 Units of Aflll

426 in $1 \times$ NEB CutSmart Buffer at $37^{\circ} \mathrm{C}$ for $1.5 \mathrm{~h}$. All reactions were stopped with $5 \mu \mathrm{L}$ Gel Loading Buffer

427 II (Life Technologies, Cat \# AM8547). Digested DNA was incubated at $75^{\circ} \mathrm{C}$ for 5 min before running 428 on 4\% (Nt.BspQl-digested samples) or 10\% (Aflll-digested samples) polyacrylamide denaturing gels.

429 Gels were dried, exposed to phosphorscreens, and imaged on a Typhoon FLA 7000 phosphorimager 430 (GE Healthcare). The frequency of collapse at each of the collapse sites (Figure 1E) was corrected for

431 the number of adenines expected for each product since products were visualized with [ $\alpha-32 \mathrm{P}] \mathrm{dATP}$. 
CGAAGAGCTGACGCGCCCTGTAGC-3' (Nt.BspQI, lagging strand samples). The template DNA was either pKV44 for leading strand analysis or pKV45 for lagging strand analysis.

\section{Preparation of nCas9 RNP complex}

439 Guide RNA was prepared by annealing AltR CRISPR-Cas9 tracrRNA, ATTO 550 (IDT) with 10-fold

(tracrRNA was limiting). The guide RNAs were then frozen at $-20^{\circ} \mathrm{C}$ until needed for experiments. The

crRNAs were designed to target specific sequences on the same strand of the $30 \mathrm{~kb}$ single molecule

min before being used in experiments.

\section{Single molecule fork collapse reactions}

The single molecule replication assay, including flow cell assembly, immunodepletion of GINS from extracts, replication reaction conditions, and image acquisition, was described in detail previously

451 (Sparks et al., 2019). Deviations from the previously published assay are described herein. Coverslips were passivated with 10\% Biotin-PEG-SVA and m-PEG-SVA MW5000 (Laysan Bio.). The buffers used to stretch DNA, wash DNA, bind nCas9, and image DNA were degassed for at least $1 \mathrm{~h}$ prior to flowing into the flow cell. Flow cells were first incubated with $0.2 \mathrm{mg} / \mathrm{mL}$ streptavidin (Sigma) for at least 15 min. Next the flow cells were washed with $500 \mu \mathrm{L}$ of DNA Blocking buffer (20 mM Tris, pH 
solution was then added at $20 \mu \mathrm{L} /$ min which contained the $2 \mathrm{nM}$ nCas9 RNP (described above) in the

462 Cas9 binding buffer. Initially, nCas9 bound specifically and nonspecifically across the entire length of

463 the DNA. Site-specifically bound Cas9 molecules remain stably associated with DNA in the presence

464 of $0.5 \mathrm{M} \mathrm{NaCl}$ (Sternberg et al., 2014). Therefore, to remove the nonspecifically bound nCas9, the

465 flow cells were washed with $60 \mu \mathrm{L}$ of Cas9 buffer containing $0.5 \mathrm{M} \mathrm{NaCl}(20 \mathrm{mM}$ Tris, $\mathrm{pH} 7.5,500 \mathrm{mM}$

$466 \mathrm{NaCl}, 5 \mathrm{mM} \mathrm{MgCl}, 1 \mathrm{mM} \mathrm{DTT}, 5 \%$ glycerol) at $20 \mu \mathrm{L} / \mathrm{min}$. The flow cells were then re-equilibrated

467 with $60 \mu \mathrm{L}$ Cas9 buffer at $20 \mu \mathrm{L} / \mathrm{min}$, before adding $30 \mu \mathrm{L}$ of $200 \mathrm{nM}$ Sytox Green (Thermo Fisher

468 Cat\# S7020) in Cas9 binding buffer at $20 \mu \mathrm{L} / \mathrm{min}$ to image the DNA and nCas9. Twenty to fifty fields of

469 view (FOVs) were imaged for both Sytox and nCas9Atto550 using alternating $488 \mathrm{~nm}$ and $561 \mathrm{~nm}$ laser

470 excitation three times ("pre-imaged DNA and nCas9"). Finally, DNA-bound sytox was washed off with

$471150 \mu \mathrm{L} 1 \times$ ELB-sucrose at $10 \mu \mathrm{L} / \mathrm{min}$. Single molecule replication experiments carried out without

472 nCas9 were prepared using the same procedure except $150 \mu \mathrm{L}$ of Cas9 binding buffer was flown into

473 the cell at $20 \mu \mathrm{L} / \mathrm{min}$ after the DNA was added, followed by flowing in the Sytox solution, imaging the

474 DNA, and flowing in the $1 x$ ELB.

Endogenous GINS was immunodepleted in two rounds from HSS and three rounds from NPE

476 (1 h each) at $4^{\circ} \mathrm{C}$. The depleted extracts were used to make licensing, initiation, and replication mixes

477 as previously described (Sparks et al., 2019) at room temperature. The double-tethered, nCas9-

478 bound DNA was licensed by flowing in $20 \mu \mathrm{L}$ of GINS-depleted HSS licensing mix at $10 \mu \mathrm{L} / \mathrm{min}$ and

479 incubating for 4-15 min. Replication was then initiated with $20 \mu \mathrm{L}$ of GINS-depleted HSS/NPE initiation

$480 \mathrm{mix}$ that included $0.01 \mathrm{mg} / \mathrm{mL}$ recombinant GINSAF647, $2 \mu \mathrm{M}$ Fen1-mKikGR D179A, and $3.7 \mathrm{nM}$

$481 \mathrm{nCas9}{ }^{A t t o 550}$ at $10 \mu \mathrm{L} / \mathrm{min}$. After $4 \mathrm{~min}, 50 \mu \mathrm{L}$ of a new GINS-depleted HSS/NPE replication mix was

482 flown in at $10 \mu \mathrm{L} / \mathrm{min}$ that included $2 \mu \mathrm{M}$ Fen1-mKikGR D179A, but did not include GINSAF647 or

483 nCas9Atto550 to remove fluorescence background from excess proteins. The absence of nCas9 in the

484 final replication mix prevents rebinding of nCas9 during the collapse reactions. Where indicated, p97-i

485 (NMS-873, Sigma) or Cul-i (MLN4924, Active Biochem) inhibitors were added to the initiation and 
replication mixes at a final concentration of $200 \mu \mathrm{M}$. Images of Fen1-mKikGR, nCas9Atto550, and

487 GINS ${ }^{\text {AF647 }}$ were acquired every minute for $1 \mathrm{~h}$ by cycling among the $488 \mathrm{~nm}\left(64-65^{\circ}\right.$ TIRF angle, 0.23

mW, 100 ms exposure, 999 EM GAIN), 561 nm (61-63 TIRF angle, 0.35 mW, 100 ms exposure, 999

EM GAIN), and $647 \mathrm{~nm}\left(61-63^{\circ}\right.$ TIRF angle, $0.15 \mathrm{~mW}, 100 \mathrm{~ms}$ exposure, 999 EM GAIN) lasers at

each of the fields of view. Specific microscope configurations were previously described (Sparks et

al., 2019). Movies were collected using NIS Elements software and saved as nd2 files.

Single molecule data analysis

494 All image analysis was performed using a combination of freely available and custom Matlab scripts.

495 Movie files were imported into a graphical user interface (GUI) using Bio-Formats to convert the nd2

496 files into readable metadata and matrices (Linkert et al., 2010). The movies were then stabilized to

497 remove drift using a Matlab script for efficient subpixel image registration (Guizar-Sicairos et al.,

498 2008). The nCas9 was targeted to the DNA asymmetrically to divide the DNA into short and long arms

499 relative to the nicking site. To determine which side of the DNA the nCas9 was binding to, the nCas9

500 channel from the "pre-imaged DNA and nCas9" (described above) with the nCas9 channel from the

501 replication movie was aligned using the same subpixel image registration script. The brightness and

502 contrast of the movies were adjusted for each channel in Matlab. The max projection of the CMG

503 signal was overlaid with the image of the unreplicated DNA (acquired before addition of extract) to

504 identify which DNA molecules were replicated. Then DNA molecules of interest were manually

505 selected to assemble kymographs. Molecules were chosen based on three criteria: 1) multiple DNA

506 molecules did not overlap significantly with each other, 2) nCas9Atto550 signal was correctly positioned

507 on the DNA, and 3) the DNA appeared to be nearly fully stretched. The kymographs were then

508 categorized according to the type of events observed, as shown in Figures S2F, S2G, and S2H. CMG

509 velocities were determined from the linear fit of the center position of the GINSAF647 signal over time.

510 These signal positions were determined using a modified version of the u-track Matlab software

511 (Jaqaman et al., 2008). The time to unload CMG after collapse was determine by counting the 
512 number of frames until GINS ${ }^{A F 647}$ signal was lost (1 min each) after nCas9Atto550 signal was lost. The

513 time to unload CMG was counted as zero minutes for the events in which CMG and nCas9 appeared

514 to be lost at the same time.

515

516 Quantification and statistical analysis

517 All ensemble experiments were repeated three or more times except for Extended Data 3b, which

518 was done twice. All single molecule experiments were performed two or more times. All image

519 analysis, including replication and sequencing gels and single molecule movies, was performed using

520 Matlab. The Kaplan-Meier curve in Figure S2E was generated using Matlab's built-in empirical

521 cumulative distribution function (ecdf). Error bars were generated from three individual biological

522 replicates. P values were calculated using Matlab's two-sample $t$-test function. Additional relevant

523 statistical details are mentioned in the figure legends.

524

\section{Code and data availability}

526 All custom-written Matlab code and raw data will be made available upon request.

\section{References}

529 Caldecott, K.W. (2008). Single-strand break repair and genetic disease. Nature Reviews Genetics 9, 530 619-631.

531 Dewar, J.M., and Walter, J.C. (2017). Mechanisms of DNA replication termination. Nature Reviews

532 Molecular Cell Biology 18, 507-516.

533 Dewar, J.M., Budzowska, M., and Walter, J.C. (2015). The mechanism of DNA replication termination 534 in vertebrates. Nature 525, 345-350.

535 Dewar, J.M., Low, E., Mann, M., Räschle, M., and Walter, J.C. (2017). CRL2Lrr1 promotes unloading of 536 the vertebrate replisome from chromatin during replication termination. Genes and Development 31, $537 \quad 275-290$.

538 Dilley, R.L., Verma, P., Cho, N.W., Winters, H.D., Wondisford, A.R., and Greenberg, R.A. (2016). Break539 induced telomere synthesis underlies alternative telomere maintenance. Nature 539, 54-58.

540 Duxin, J.P., Dewar, J.M., Yardimci, H., and Walter, J.C. (2014). Repair of a DNA-protein crosslink by

541 replication-coupled proteolysis. Cell 159, 349-357. 
542 Fu, Y. v., Yardimci, H., Long, D.T., Ho, V., Guainazzi, A., Bermudez, V.P., Hurwitz, J., van Oijen, A., 543 Sch??rer, O.D., Walter, J.C., et al. (2011). Selective bypass of a lagging strand roadblock by the 544 eukaryotic replicative DNA helicase. Cell 146, 931-941.

545 Guizar-Sicairos, M., Thurman, S.T., and Fienup, J.R. (2008). Efficient subpixel image registration 546 algorithms.

547 Haber, J.E. (1999). DNA recombination: The replication connection. Trends in Biochemical Sciences

$548 \quad 24,271-275$.

549 Hakem, R., de La Pompa, J.L., Sirard, C., Mo, R., Woo, M., Hakem, A., Wakeham, A., Potter, J., 550 Reitmair, A., Billia, F., et al. (1996). The tumor suppressor gene Brca1 is required for embryonic 551 cellular proliferation in the mouse. Cell 85, 1009-1023.

552 Hashimoto, Y., Puddu, F., and Costanzo, V. (2012). RAD51- and MRE11-dependent reassembly of 553 uncoupled CMG helicase complex at collapsed replication forks. Nature Structural \& Molecular 554 Biology 19, 17-24.

555 Hengel, S.R., Spies, M.A., and Spies, M. (2017). Small-Molecule Inhibitors Targeting DNA Repair and DNA Repair Deficiency in Research and Cancer Therapy. Cell Chemical Biology 24, 1101-1119. Hogg, M., Osterman, P., Bylund, G.O., Ganai, R.A., Lundström, E.B., Sauer-Eriksson, A.E., and Johansson, E. (2014). Structural basis for processive DNA synthesis by yeast DNA polymerase É. Nature Structural and Molecular Biology 21, 49-55.

560 Ira, G., and Haber, J.E. (2002). Characterization of RAD51-Independent Break-Induced Replication 561 That Acts Preferentially with Short Homologous Sequences. Molecular and Cellular Biology 22, 63845626392.

563 Jakobsen, K.P., Nielsen, K.O., Løvschal, K. v, Rødgaard, M., Andersen, A.H., and Correspondence, L.B. (2019). Minimal Resection Takes Place during Break-Induced Replication Repair of Collapsed Replication Forks and Is Controlled by Strand Invasion. CellReports 26, 836-844.e3. Jaqaman, K., Loerke, D., Mettlen, M., Kuwata, H., Grinstein, S., Schmid, S.L., and Danuser, G. (2008). Robust single-particle tracking in live-cell time-lapse sequences. Nature Methods 5, 695-702. Jinek, M., Chylinski, K., Fonfara, I., Hauer, M., Doudna, J.A., and Charpentier, E. (2012). A programmable dual-RNA-guided DNA endonuclease in adaptive bacterial immunity. Science 337, $570816-821$. Kang, Y.H., Galal, W.C., Farina, A., Tappin, I., and Hurwitz, J. (2012). Properties of the human Cdc45/Mcm2-7/GINS helicase complex and its action with DNA polymerase $\varepsilon$ in rolling circle DNA synthesis. Proceedings of the National Academy of Sciences of the United States of America 109, 6042-6047.

Langston, L., and O'Donnell, M. (2017). Action of CMG with strand-specific DNA blocks supports an internal unwinding mode for the eukaryotic replicative helicase. ELife 6.

Lebofsky, R., Takahashi, T., and Walter, J.C. (2009). DNA replication in nucleus-free xenopus egg extracts. Methods in Molecular Biology 521, 229-252.

Linkert, M., Rueden, C.T., Allan, C., Burel, J.M., Moore, W., Patterson, A., Loranger, B., Moore, J., Neves, C., MacDonald, D., et al. (2010). Metadata matters: Access to image data in the real world. Journal of Cell Biology 189, 777-782.

Loveland, A.B., Habuchi, S., Walter, J.C., and van Oijen, A.M. (2012). A general approach to break the concentration barrier in single-molecule imaging. Nature Methods 9, 987-992.

Lydeard, J.R., Lipkin-Moore, Z., Sheu, Y.-J., Stillman, B., Burgers, P.M., and Haber, J.E. (2010). Breakinduced replication requires all essential DNA replication factors except those specific for pre-RC assembly. Genes \& Development 24, 1133-1144. 
Mayle, R., Campbell, I.M., Beck, C.R., Yu, Y., Wilson, M., Shaw, C.A., Bjergbaek, L., Lupski, J.R., and Ira, G. (2015). Mus81 and converging forks limit the mutagenicity of replication fork breakage. Science 349, 742-747. Nacson, J., Marcantonio, D. di, Wang, Y., Sykes, S.M., Johnson, N., Nacson, J., Marcantonio, D. di, Wang, Y., Bernhardy, A.J., Clausen, E., et al. (2020). BRCA1 Mutational Complementation Induces Synthetic Viability Short Article BRCA1 Mutational Complementation Induces Synthetic Viability. Molecular Cell 1-9. (2017). Acute inactivation of the replicative helicase in human cells triggers MCM8-9-dependent DNA synthesis. Genes and Development 31, 816-829. and Bradley, A. (1997). Embryonic lethality and radiation hypersensitivity mediated by Rad51 in mice lacking Brca2. Nature 386, 804-810. Sonneville, R., Bhowmick, R., Hoffmann, S., Mailand, N., Hickson, I.D., and Labib, K. (2019). TRAIP drives replisome disassembly and mitotic DNA repair synthesis at sites of incomplete DNA replication. ELife 8. Yamaguchi-Iwai, Y., Takeda, S., and Chair, B. (1998). Rad51-deficient vertebrate cells accumulate chromosomal breaks prior to cell death. Helicase Bypasses DNA-Protein Cross-Links to Facilitate Their Repair. Cell 176, 167-181. the CRISPR RNA-guided endonuclease Cas9. Nature 507, 62-67. Topoisomerase I Cleavage Complexes on the Leading Strand of Ribosomal DNA into 5'Phosphorylated DNA Double-Strand Breaks by Replication Runoff. Molecular and Cellular Biology 20, 3977-3987.

Tsuzuki, T., Fujii, Y., Sakumi, K., Tominaga, Y., Nakao, K., Sekiguchi, M., Matsushiro, A., Yoshimura, Y., and Morita, T. (1996). Targeted disruption of the Rad51 gene leads to lethality in embryonic mice. Proceedings of the National Academy of Sciences of the United States of America 93, 6236-6240. Vilenchik, M.M., and Knudson, A.G. (2003). Endogenous DNA double-strand breaks: Production, fidelity of repair, and induction of cancer. Proceedings of the National Academy of Sciences of the United States of America 100, 12871-12876.

Walter, J.C., Sun, L., and Newport, J. (1998). Regulated chromosomal DNA replication in the absence of a nucleus. Molecular Cell 1, 519-529.

Wang, A.S., Chen, L.C., Wu, R.A., Hao, Y., McSwiggen, D.T., Heckert, A.B., Richardson, C.D., Gowen, B.G., Kazane, K.R., Vu, J.T., et al. (2020). The histone chaperone FACT induces Cas9 multi-turnover behavior and modifies genome manipulation in human cells. Molecular Cell In Press.

Wasserman, M.R., Schauer, G.D., O’Donnell, M.E., and Liu, S. (2019). Replication Fork Activation Is Enabled by a Single-Stranded DNA Gate in CMG Helicase. Cell 178, 600-611.e16. et al. (2013). Pif1 helicase and Pol $\delta$ promote recombination-coupled DNA synthesis via bubble migration. Nature 502, 393-396. 

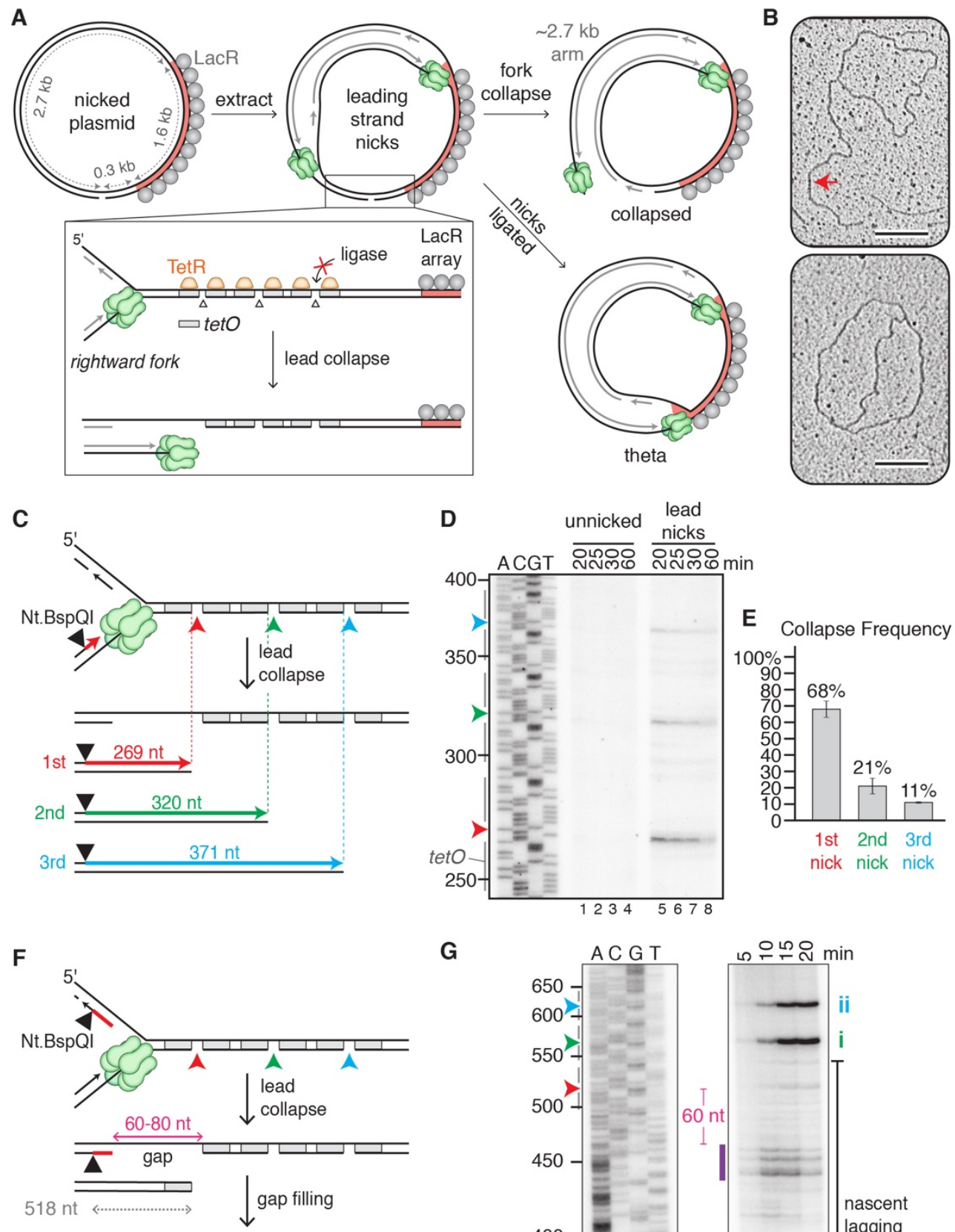

G
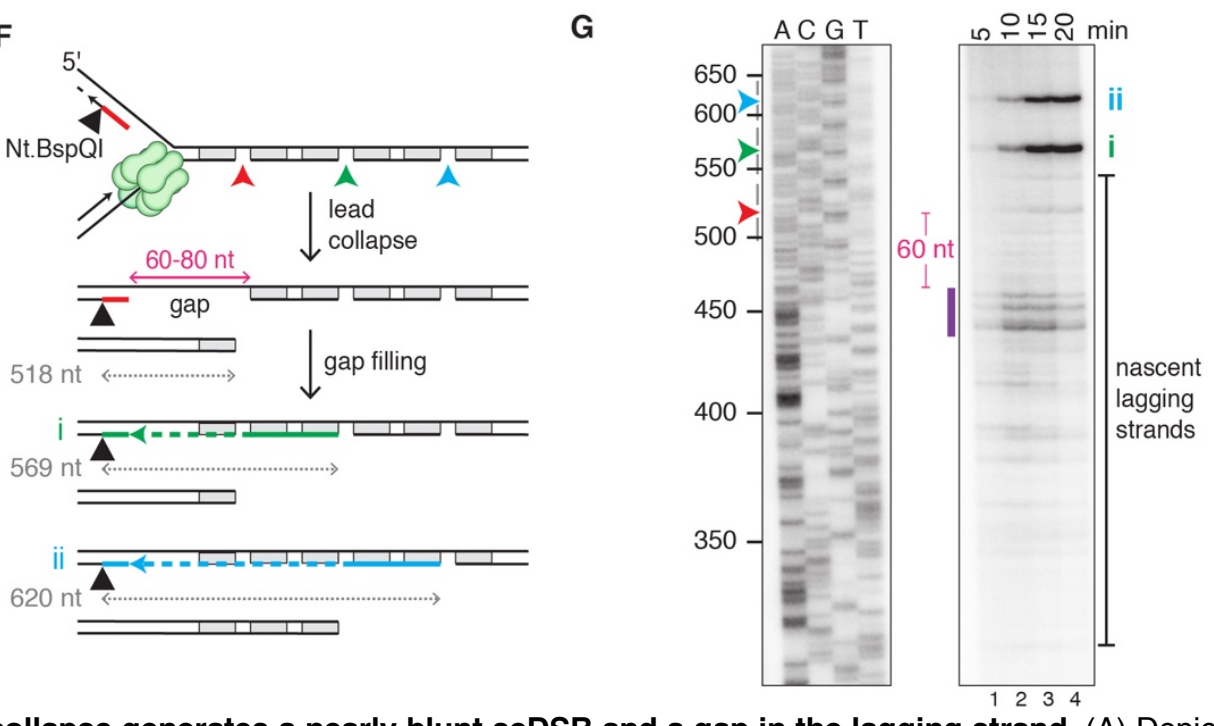

Figure 1. Lead collapse generates a nearly blunt seDSB and a gap in the lagging strand. (A) Depiction of experimental approach. (B) TEM images of collapsed (top) and theta (bottom) structures. Red arrow, $2.7 \mathrm{~kb}$ arm. Scale bar, $200 \mathrm{~nm}$. (C and D) Depiction (C) and analysis (D) of nascent leading strands generated after lead collapse and DNA digestion with Nt.BspQI. Gray bars in (D) denote tetO sites. The DNA was visualized by autoradiography, and only the relevant segment of the gel is shown. (E) Percentage collapse at each of the three nicks out of total collapse events. Error bars, standard deviations from three independent experiments. (F) Repeat of (D), with Nt.BspQI site located on nascent lagging strand. After fork collapse at the first nick, gap filling from the 3 ' end of the nick to the final Okazaki fragment and ligation should create a $569 \mathrm{nt}$ product (i, green line). Collapse at the second nick, gap filling, and ligation should generate a $620 \mathrm{nt}$ product (ii, blue line). (G) Nascent lagging strand analysis of products generated after lead collapse. Purple bar, most prominent lagging strand products. 
A i) unnicked
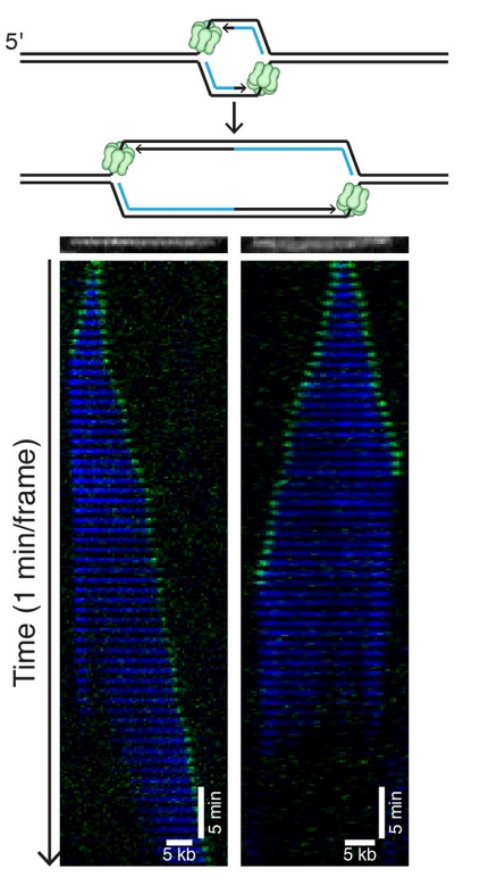

ii) nCas9 - lead collapse
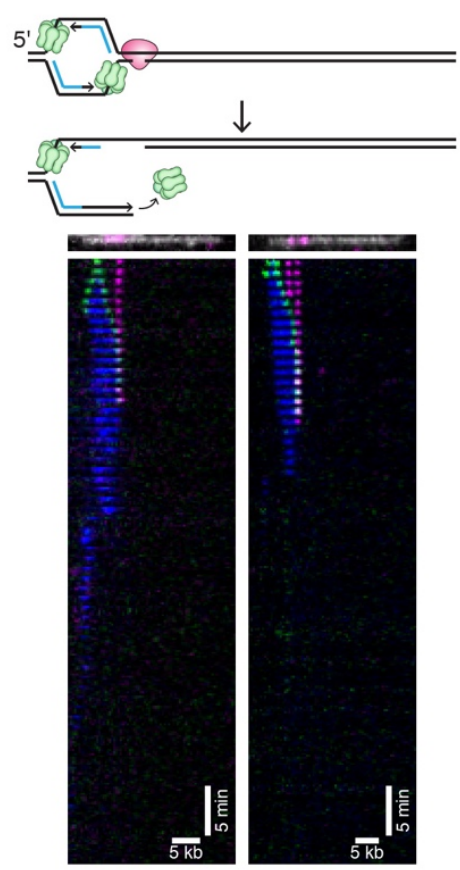

iii) dCas9 - no collapse

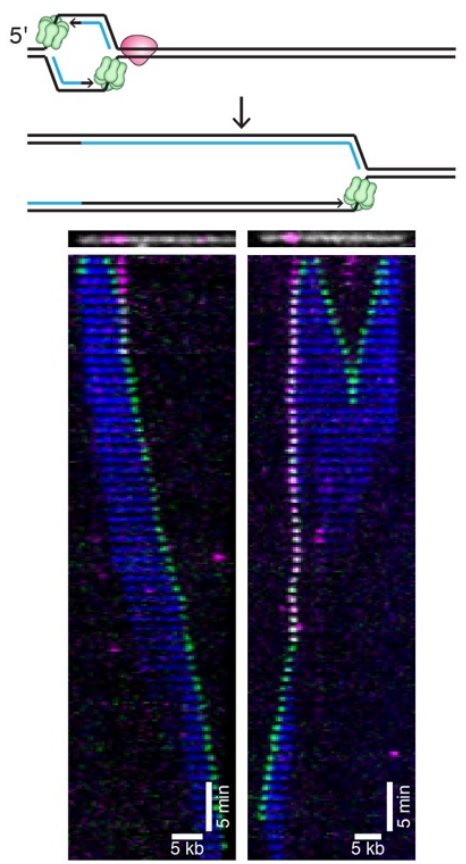

644

645

646

647

648

649

650

651

652

653

654

655

656

657

658

659

660

B

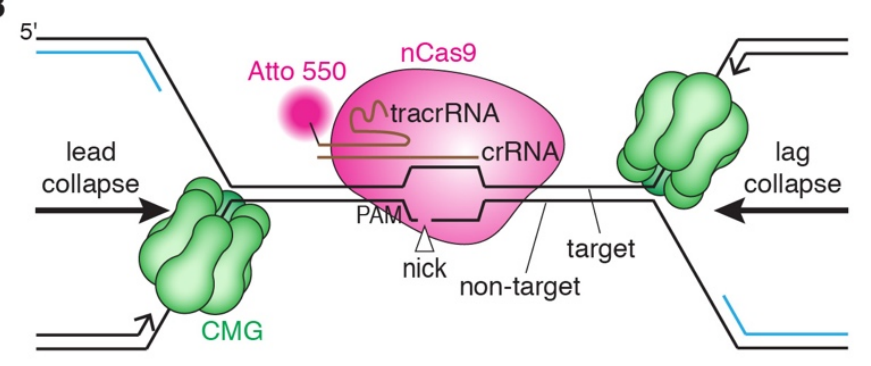

Figure 2. CMG is lost at the nick after lead collapse. (A) Representative single molecule kymographs and interpretive cartoons of i) replication on unnicked DNA, ii) lead collapse on nCas9-nicked DNA (note that in the kymograph on the right, the left nCas9 appears to dissociate before CMG arrival), and iii) replication fork encounter with dCas9. CMG is shown in green, Fen1mKikGR in blue, nCas9 in magenta. Although more than one Cas9 is frequently bound, only one is depicted in cartoons for simplicity. Kymographs were created by stacking the frames of a movie (one-minute intervals). The DNA (white) and nCas9 (magenta) shown above each kymograph are from imaging the nCas9 and DNA before extract addition. See also Supplemental Videos 1-3. (B) Schematic of nCas9 H840A bound to DNA with replication forks arriving from the left (lead collapse) or right (lag collapse). 


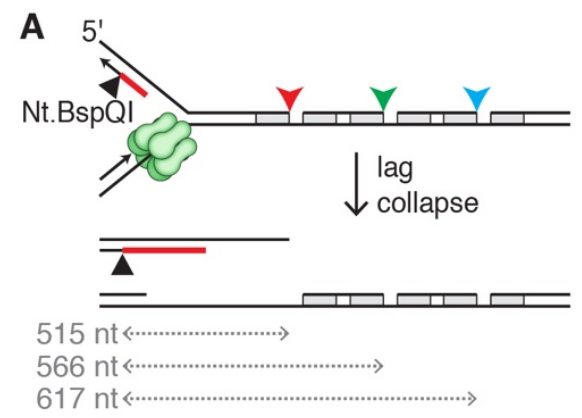

B

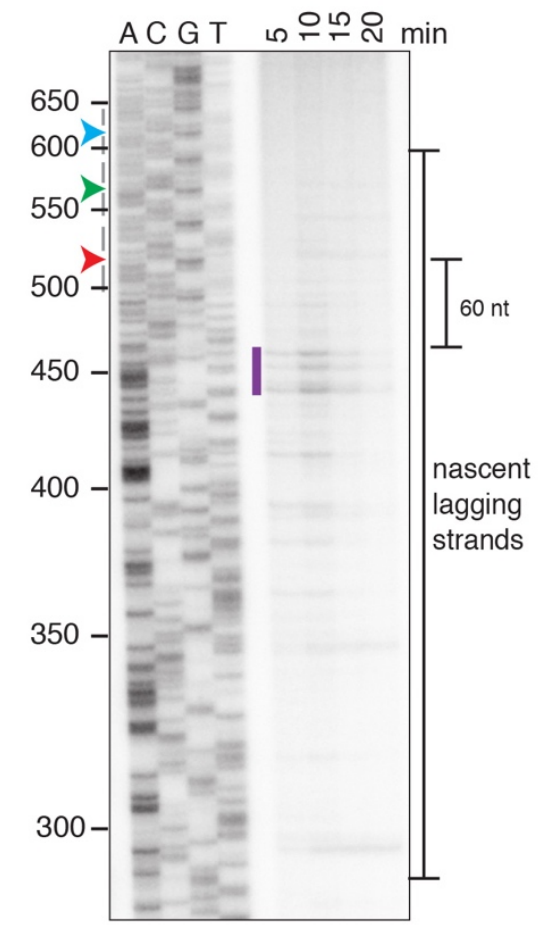

C
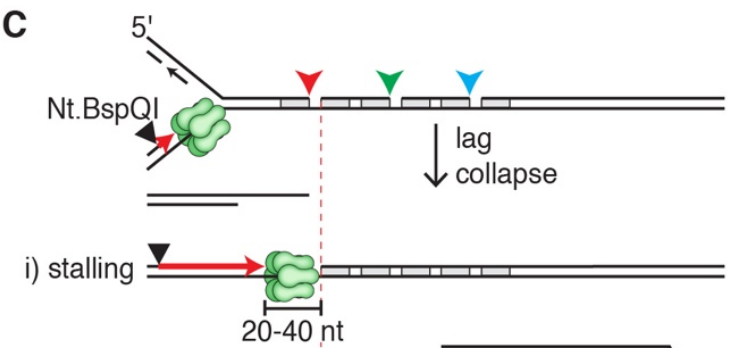

ii) unwinding

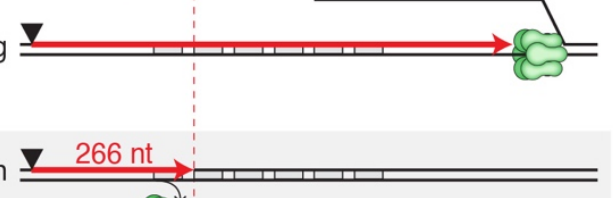

iv) translocation

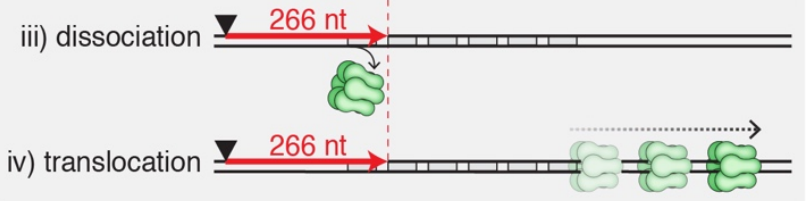

\section{D}

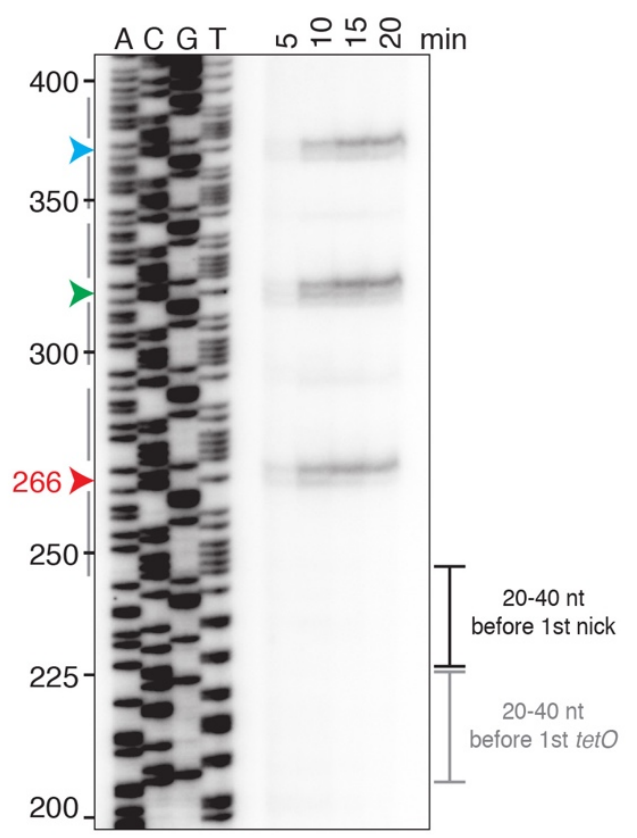

Figure 3. Lag collapse generates a seDSB with a 3' ssDNA overhang. (A) Depiction of nascent lagging strand products generated from lag collapse at the first nick. $515 \mathrm{nt}, 566 \mathrm{nt}$, and $617 \mathrm{nt}$ correspond to the distances between the Nt.BspQI site and the three nicks. (B) Analysis of lagging strand products after lag collapse and DNA digestion with Nt.BspQI. Arrowheads, location of nicks. (C) Four different CMG fates after lagging strand fork collapse, including predicted size of the leading strand. Text for details. (D) Analysis of leading strand products after lag collapse and DNA digestion with Nt.BspQI. Black bracket, location where leading strands would stall in model (C, i). The lack of signal 20-40 nt before the $1^{\text {st }}$ tetO site (gray bracket) indicates that CMG does not stall at TetR. 
A
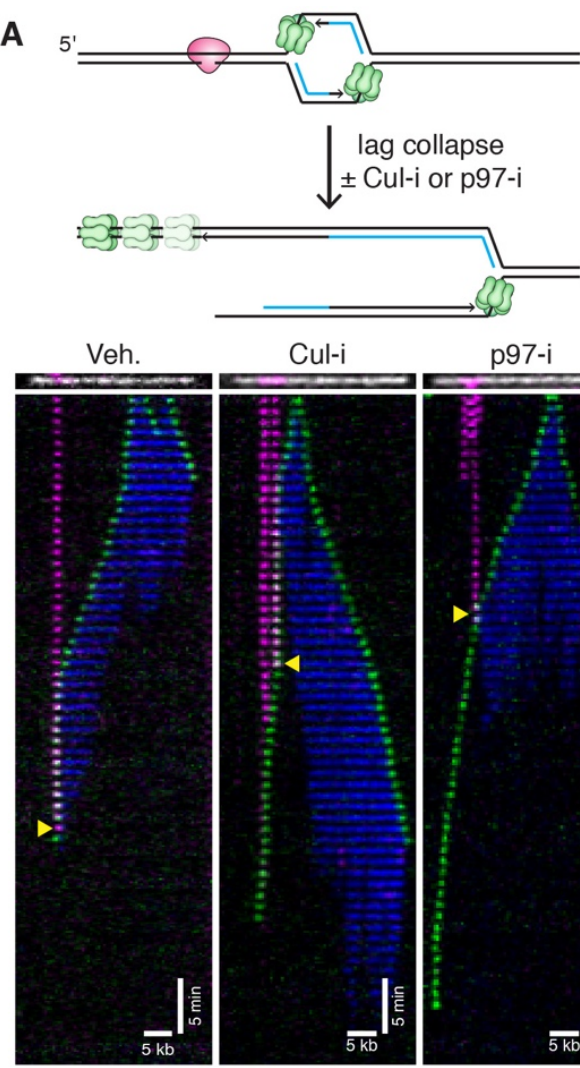

$\downarrow \begin{aligned} & \text { lag collapse } \\ & \pm \text { Cul-i or } p 97-\mathrm{i}\end{aligned}$

$5 \mathrm{~kb}$

D
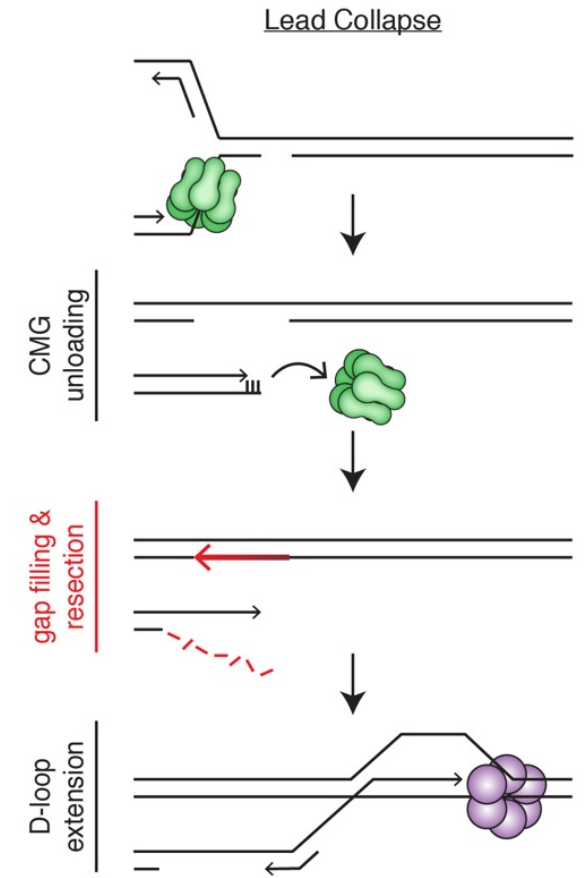

B

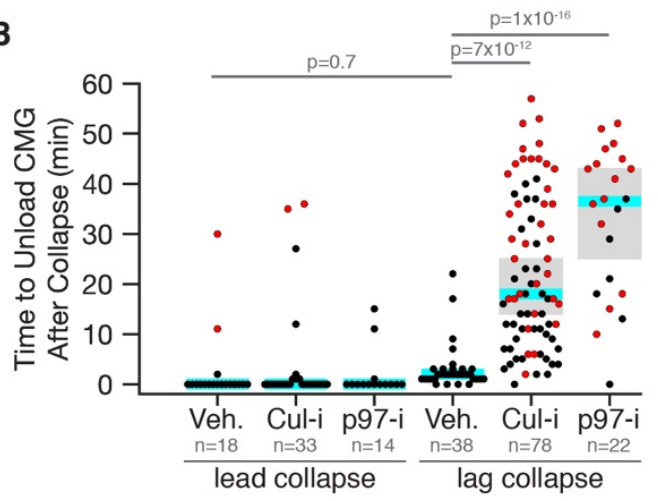

C

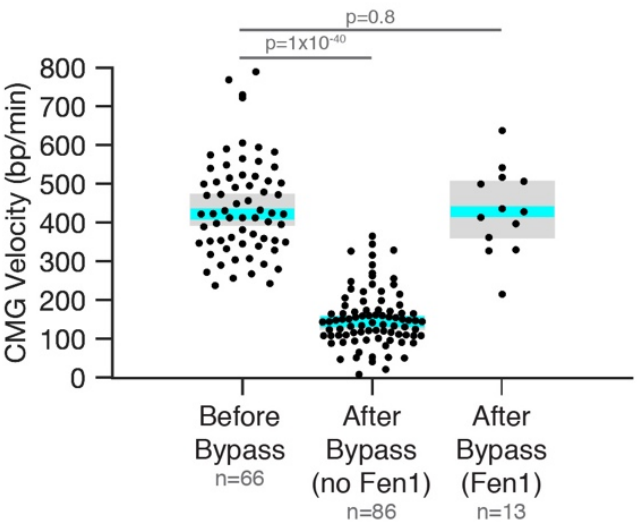

Lag Collapse

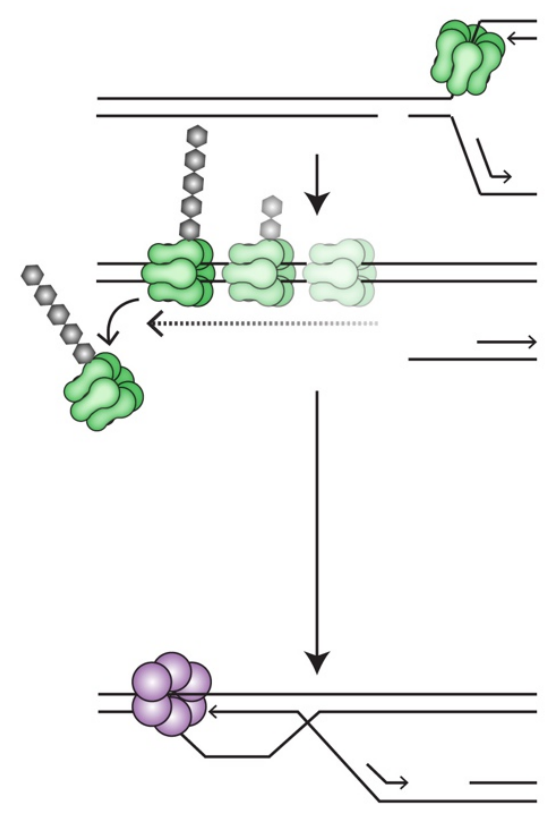

Figure 4. Active CMG unloading from double-stranded DNA during lag collapse. (A) Representative single molecule kymographs and interpretive cartoons when $\mathrm{CMG}$ approaches $\mathrm{nCas} 9$ from the long arm in the indicated conditions. Yellow triangle, moment of collapse (nCas9 loss). The DNA (white) and nCas9 (magenta) shown above each kymograph are from imaging the nCas9 and DNA before extract addition. See also Supplemental Videos 4-6. Note that in the middle kymograph, the left nCas9 appears to dissociate before arrival of CMG. (B) Distribution of CMG unloading times after lead and lag collapse in the indicated conditions. Because many CMGs persisted until the end of the 60 min experiment (red circles), the median times reported are lower limits. In (B) and (C), blue line is the median, and gray box represents the $95 \%$ confidence for the median determined from bootstrapping analysis. (C) Distribution of CMG velocities before and after nCas9 bypass with and BIR. 
bioRxiv preprint doi: https://doi.org/10.1101/2020.08.17.254235; this version posted August 17, 2020. The copyright holder for this preprint (which was not certified by peer review) is the author/funder. All rights reserved. No reuse allowed without permission.

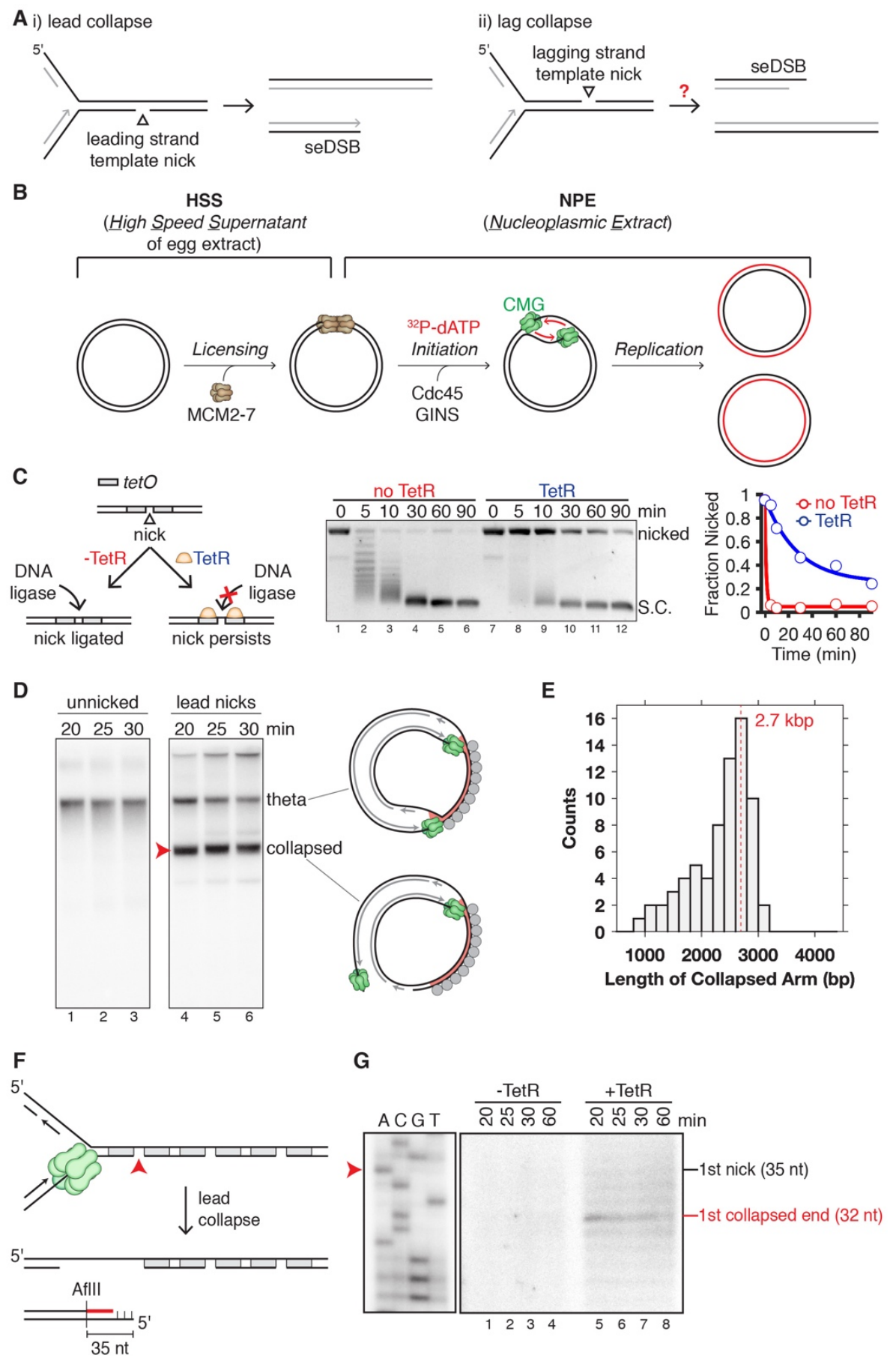

Figure S1. Leading strand fork collapse in Xenopus extracts using TetR-nick protection strategy. (A) General representations of lead and lag fork collapse. (B) Experimental workflow for DNA replication in Xenopus egg extracts. DNA is first licensed in HSS to load the MCM2-7 double hexamer, followed by addition of NPE to initiate a single round of DNA replication. (C) TetR-nick protection strategy. In the absence of TetR, nicks are ligated by DNA ligase (left branch of cartoon, lanes 1-6, and red line in graph). In the presence of TetR, nicks are much more stable, presumably because TetR prevents access of DNA ligases to the nicks (right branch of cartoon, lanes 7-12, and blue line in graph). (D) Unnicked plasmid or plasmid with a bottom strand nick (Lead collapse) was replicated in the presence of TetR and LacR using egg extracts (depicted in Figure $1 \mathrm{~A})$. (E) Length distribution of the collapsed arms visualized by EM. The theoretical length of the collapsed arm after lead collapse is $2.7 \mathrm{kbp}$. Length is estimated assuming B-form DNA with a rise per basepair of $0.34 \mathrm{~nm}$. (F) Schematic of the nascent leading strand products generated from lead collapse at the first nick. The red line depicts the newly synthesized leading strand after digestion by Aflll, which cuts $35 \mathrm{nt}$ from the nick. (G) Plasmid with bottom strand nick (lead collapse) was replicated in egg extract in the presence and absence of TetR, as indicated. Nascent leading strand products were analyzed on a urea PAGE gel after lead collapse and Aflll digestion. In the absence of TetR, nicks are ligated, explaining the lack of collapse products. The prominent leading strand product $(32 \mathrm{nt})$ is three $\mathrm{nt}$ shorter than the terminal Aflll fragment ( $35 \mathrm{nt})$. 

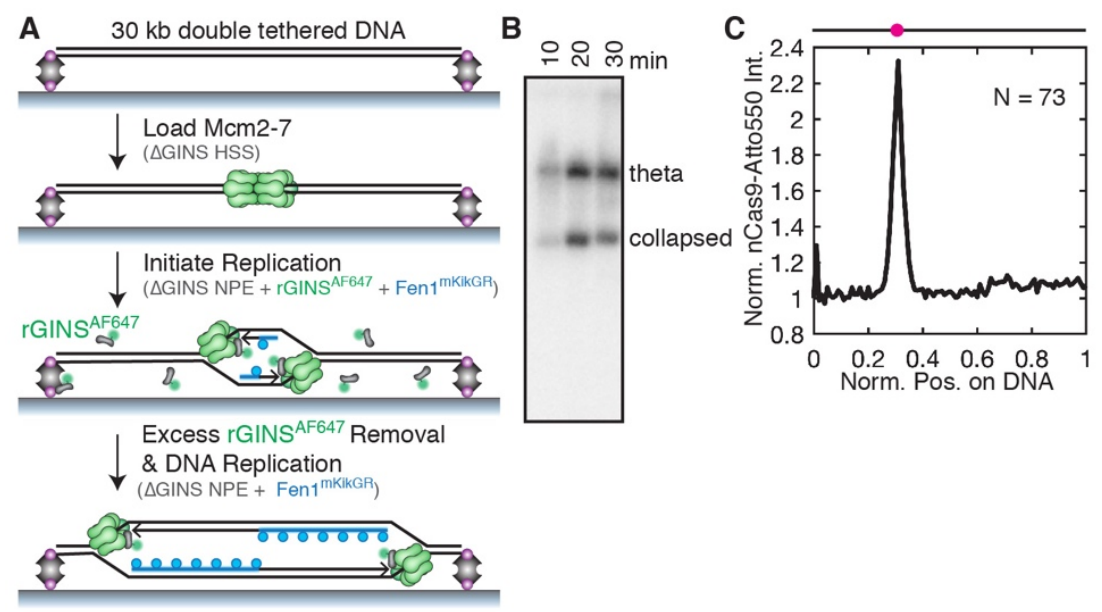
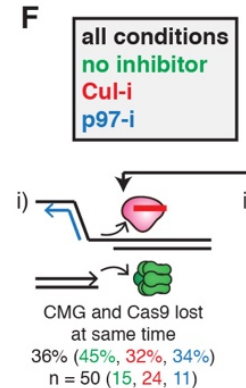

ii)

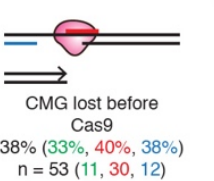

Lead Collapse

$n=140(33,75,32)$

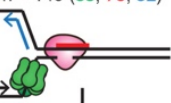

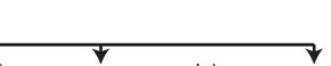

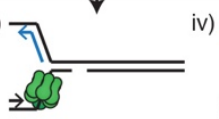

CMG persists longer than Cas9 $25 \%(21 \%, 28 \%, 22 \%)$ $\mathrm{n}=35(7,21,7)$
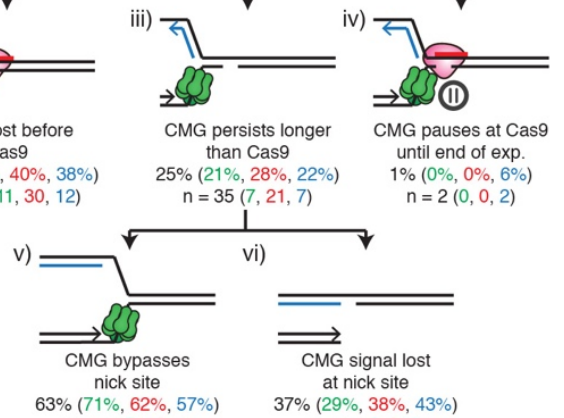

vi)
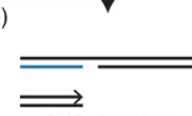

CMG signal los at nick site

$63 \%(71 \%, 62 \%, 57 \%) \quad 37 \%(29 \%, 38 \%, 43 \%)$

$n=22(5,13,4)$

$n=13(2,8,3)$ vii)
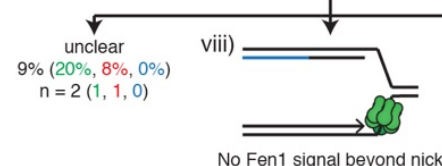
$9 \%(20 \%, 8 \%, 0 \%)$
No Fen1 signal beyond nick
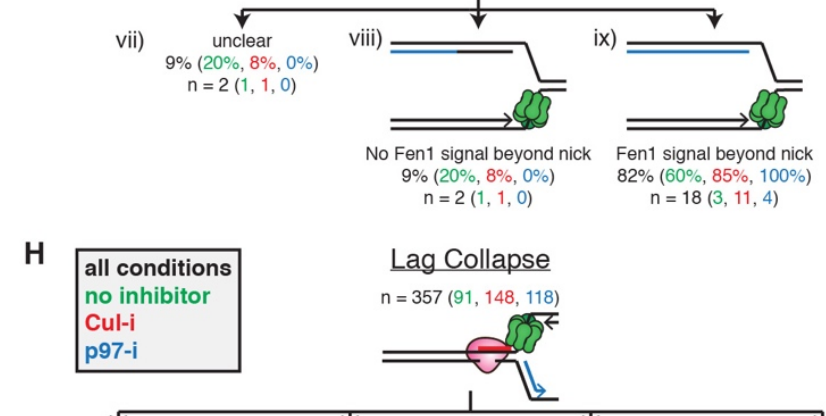

i)

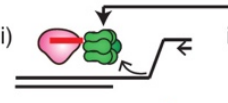

CMG and Cas9 lost
at same time at same time $2 \%(4 \%, 1 \%, 1 \%)$
$n=6(4,1,1)$

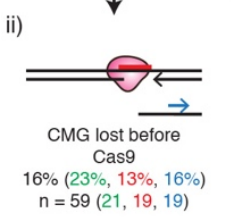

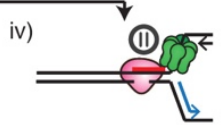

CMG pauses at Cas 9 until end of exp. $\mathrm{n}=100(12,17,71)$

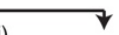

iii)

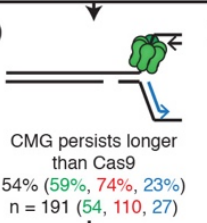

v)
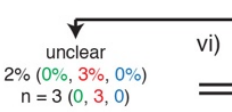

$\mathrm{n}=191(54,11$
G
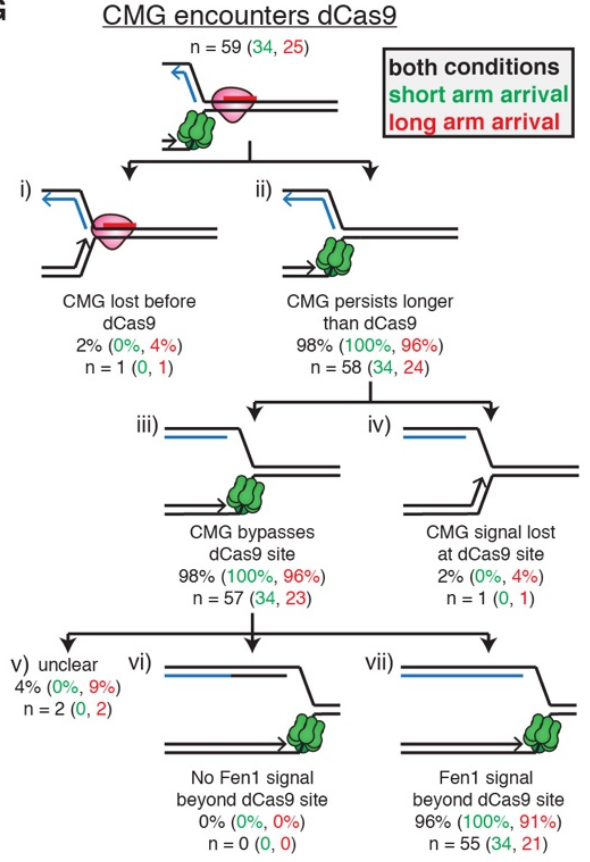
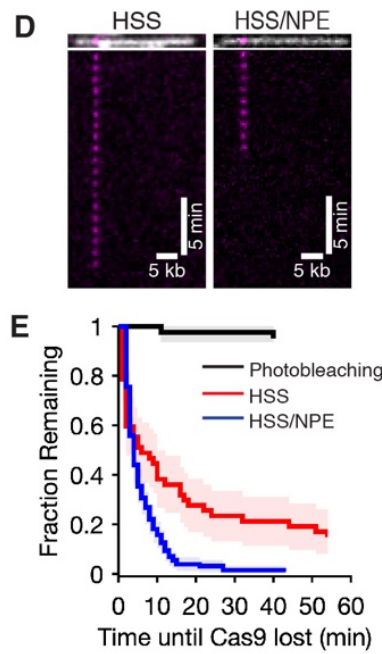

\section{(n)}


bioRxiv preprint doi: https://doi.org/10.1101/2020.08.17.254235; this version posted August 17, 2020. The copyright holder for this preprint (which was not certified by peer review) is the author/funder. All rights reserved. No reuse allowed without permission.

698

699

700

701

702

703

704

705

706

707

708

709

710

711

712

713

714

715

716

717

718

719

720
Figure S2. Single molecule visualization of CMG and nCas9 on double-tethered DNA. (A) Schematic of single molecule replication assay. First, double-biotinylated $30 \mathrm{~kb}$ DNA is stretched and double-tethered to a streptavidin-coated coverslip. Next, GINS-depleted HSS is injected into the flow cell to load MCM2-7 double hexamer (licensing). Replication is then initiated with GINS-depleted NPE supplemented with recombinant GINS tagged with Alexa Fluor 647 (rGINSAF647, gray shape with green circle) and Fen1mKikGR (blue circle). After 4 minutes, excess GINS is removed by flowing in GINS-depleted NPE supplemented only with Fen1 1 kikGR. Fen1 ${ }^{\mathrm{mkikGR}}$ delays the dissociation of PCNA from DNA and therefore appears to bind PCNA molecules that are deposited during Okazaki fragment synthesis, leading to labeling of the entire replication bubble. (B) nCas9-induced replication fork collapse. The experiment is similar to the one shown in Figure S1D, except that nCas9 H840A is used to nick the DNA. (C) Position of nCas9Atto550 signal on double-tethered DNA. The black line above the plot shows the theoretical position for nCas9 binding (magenta dot). Only a single nCas9 was targeted to the DNA for this experiment. (D) Representative kymographs of nCas9 binding to DNA in HSS or 1:2 mixtures of HSS and NPE which mimic the condition used during single molecule DNA replication. The DNA (white) and nCas9 (magenta) shown above each kymograph are from imaging the nCas9 and DNA before adding the extracts. (E) The rates of nCas9 loss in HSS or HSS/NPE compared to the photobleaching rate. Shaded regions represent $90 \%$ confidence bounds. $(F, G$, and $H)$ Breakdown of all categories of events observed during $(F)$ lead collapse, $(G)$ CMG collision with dCas9, or $(H)$ lag collapse in single molecule assays. Below each category, the number of events observed in that category under three different conditions is listed in brackets. For $F$ and $H$, no drug, green; Cul-i, red; p97-i, blue. For G, CMG collides with dCas9 from the short arm, green; CMG collides with dCas9 from the long arm, red. Black, sum of all conditions in a category. Cartoons only depict arrival from the short arm. Percentages are calculated for each tier of the hierarchy. 
A

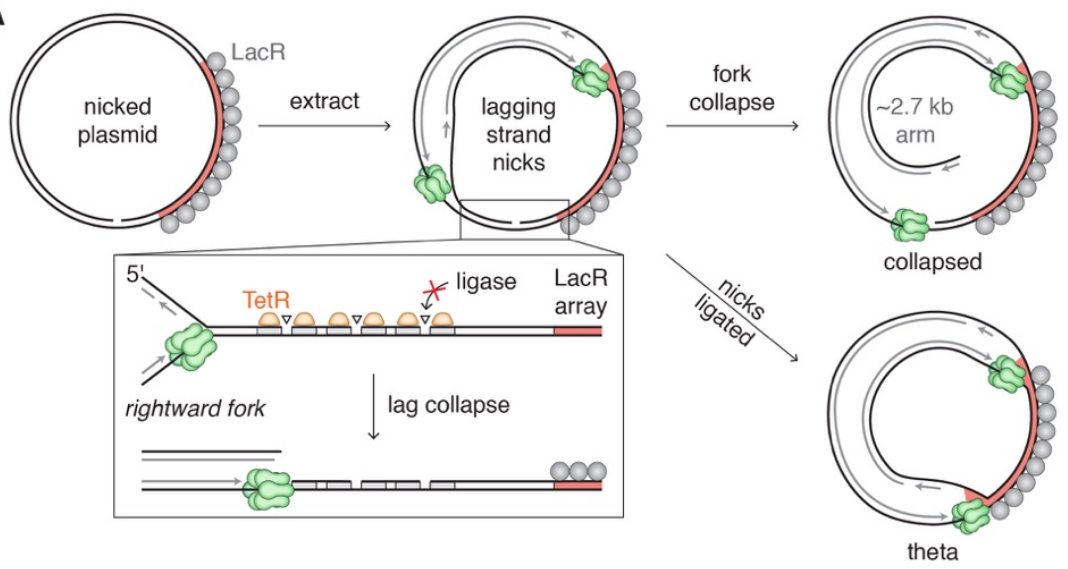

B
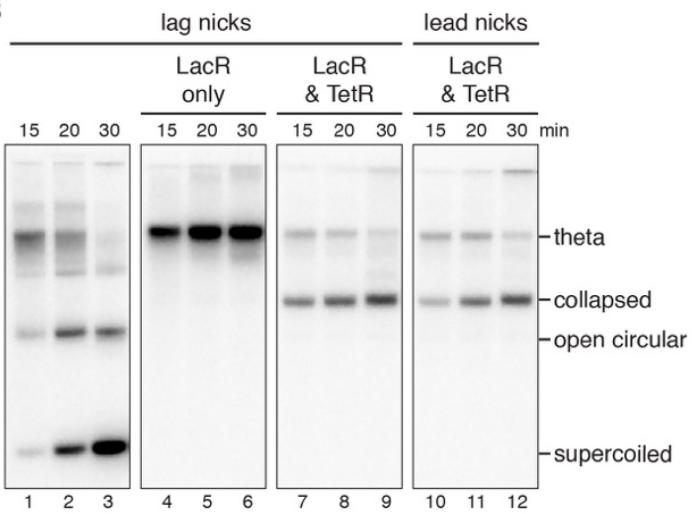

C

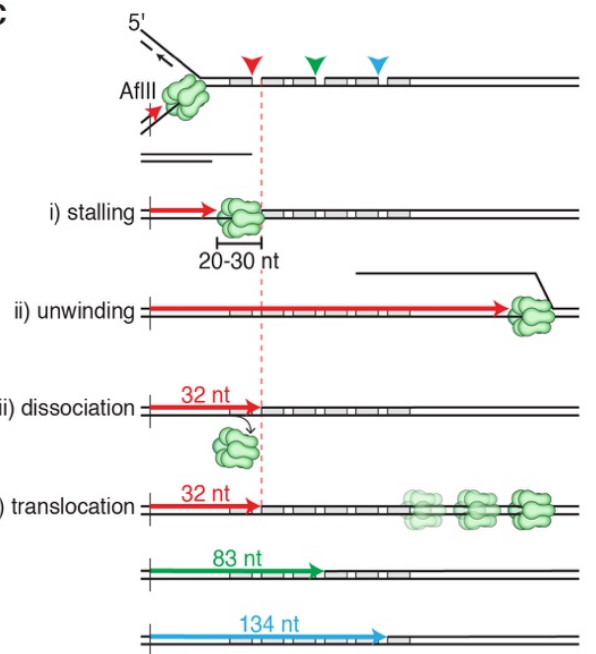

D

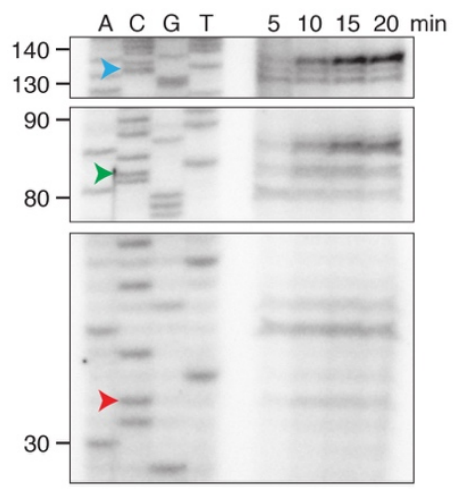

Figure S3. Lagging strand fork collapse in Xenopus extracts using the Tet-nick strategy. (A) Depiction of structures generated during replication of a top strand (lag collapse) plasmid in the presence of TetR. Inset, detailed representation of the three nicks and flanking tetO sites. (B) Plasmid with a bottom strand (lead) nick or top strand (lag) nick was replicated in the presence of LacR +/-TetR using egg extracts. In the absence of TetR and LacR (lanes 1-3), nicks were ligated, and replication went to completion, generating the expected open circular and supercoiled products. In the presence of LacR, but no TetR (lanes 4-6), the unprotected nicks were ligated, and a prominent theta band was generated from forks converging on the LacR array (as in (A), bottom route). In the presence of both LacR and TetR (lanes 7-9), the nicks were protected, and lag collapse occurred (as in (A), top route), generating the same collapsed product detected from lead collapse (lanes 10-12 and Figure S1D). (C) Same as Figure 3B, except that lead products for all three nicks are shown for model iv. (D) Same as Figure 3D, except that DNA was digested with Aflll (35 nt away from first nick) and run on a 10\% polyacrylamide gel to improve the resolution. A significant fraction of the leading strands were extended approximately $3 \mathrm{nt}$ beyond the nick site, consistent with limited strand displacement synthesis. 
A

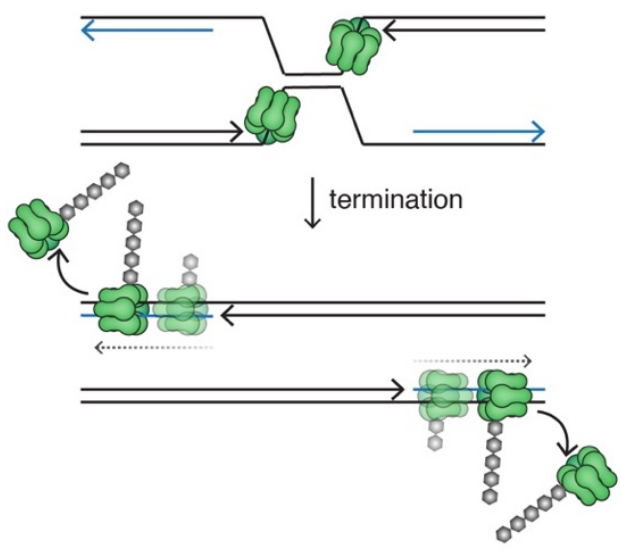

B
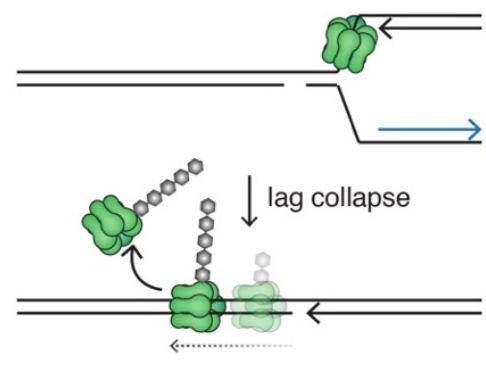

C
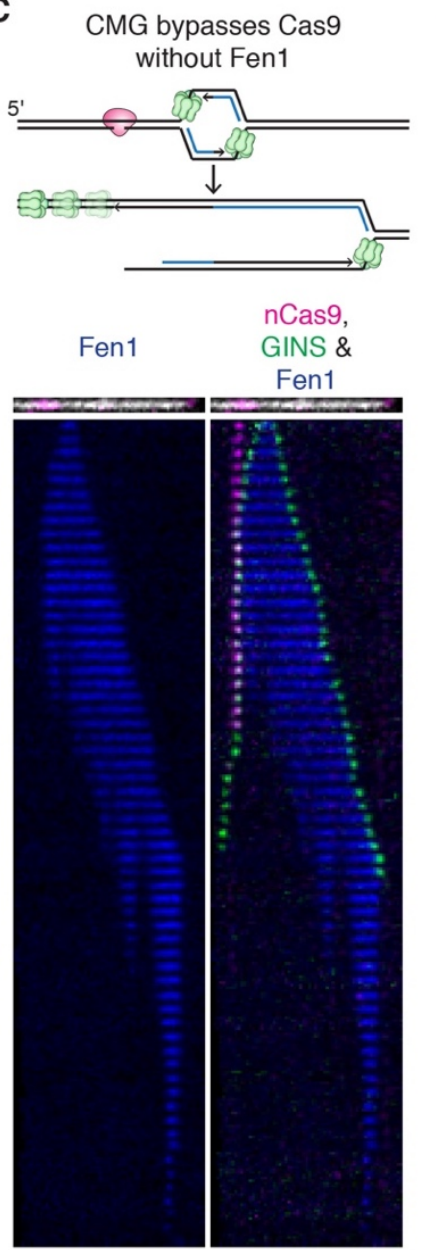

D

Unclear if CMG bypasses Cas9 without Fen1

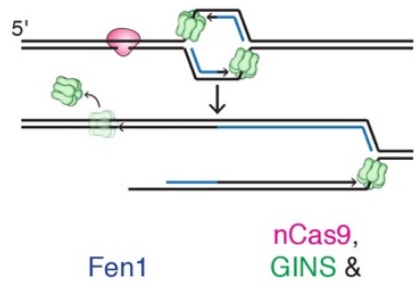

Fen1

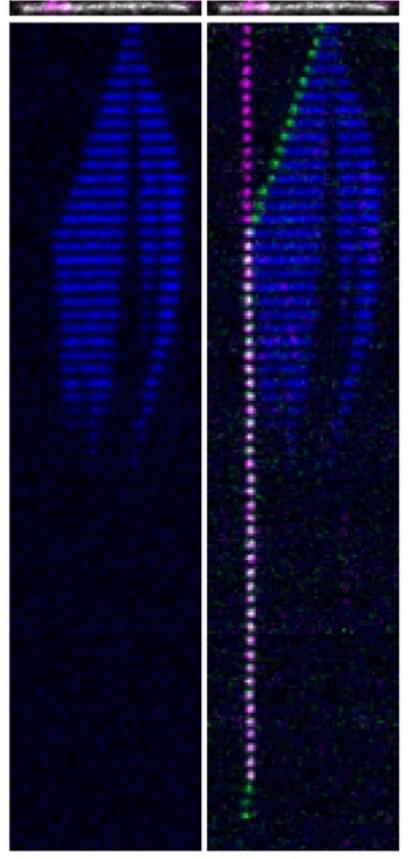

E

CMG bypasses Cas 9 with Fen1

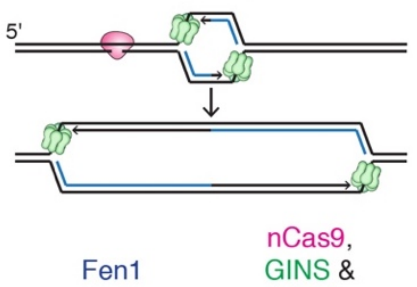

Fen1

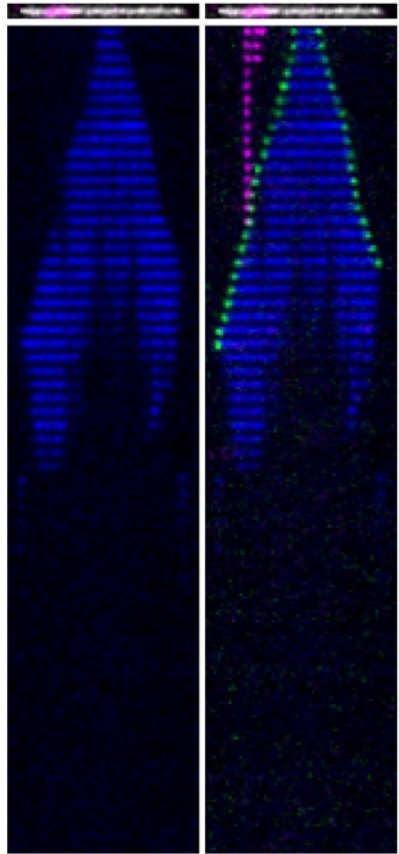

Figure S4. Parallels between termination and collapse, and representative kymographs of different classes of "lag collapse" events. (A) During replication termination, replication forks converge, CMGs pass each other and pass onto dsDNA when they hit the nascent lagging strand of the opposing fork, followed by CRL2LRR1-dependent CMG ubiquitylation and extraction by the p97 ATPase (Dewar et al., 2017). (B) During lag collapse, CMG passes onto dsDNA when it hits the nick in the lagging strand template, followed by cullin-RING and p97-dependent chromatin extraction. (C) Representative single molecule kymograph and interpretive cartoon of lag collapse events in which CMG translocated unidirectionally away from the collapse site after collapse (quantified in Figure S2H,ix). The DNA (white) and nCas9 (magenta) shown above each kymograph are from imaging the nCas9 and DNA before extract addition. (D) Representative kymograph of lag collapse events in which CMG was unloaded too rapidly to assess whether Fen1 signal trailed behind CMG (quantified in Figure S2H,viii). (E) Representative kymograph of events in which CMG bypassed nCas9 with a trailing Fen1 signal. This signature suggests that the fork did not collapse, presumably because nCas9 did not nick the DNA or because the nick was ligated prior to CMG encounter (quantified in Figure $\mathrm{S} 2 \mathrm{H}, \mathrm{x}$ ). 


\section{Supplemental Information}

Supplemental Video 1. Replication of unnicked DNA. Replication of a single, double tethered DNA was visualized by

TIRF microscopy. CMG shown in green, Fen1mkikGR in blue. Frames collected every minute for $1 \mathrm{~h}$. Corresponding kymograph in Figure 2A,i.

754

Supplemental Video 2. Leading strand fork collapse. Collision of a replication fork arriving from the short arm with $\mathrm{nCas} 9$ results in leading strand fork collapse. CMG shown in green, Fen1 1 mKikGR in blue, nCas9 in magenta. Frames collected every minute for $1 \mathrm{~h}$. Corresponding kymograph in Figure 2A,ii.

758

Supplemental Video 3. Replication collision with dCas9. Unlike leading strand fork collapse, in which CMG is lost at the nCas9, CMG bypasses dCas9 and replication continues. CMG shown in green, Fen1 ${ }^{\mathrm{mkikGR}}$ in blue, dCas9 in magenta. Frames collected every minute for $1 \mathrm{~h}$. Corresponding kymograph in Figure 2A,iii.

762

Supplemental Video 4. Lagging strand fork collapse. Collision of a replication fork arriving from the long arm with nCas9 results in lagging strand fork collapse. CMG shown in green, Fen1 ${ }^{\mathrm{mkikGR}}$ in blue, dCas9 in magenta. Frames collected every minute for $1 \mathrm{~h}$. Corresponding kymograph in Figure 4A, Veh.

766

767

Supplemental Video 5. Lag collapse in the presence of Cul-i. CMG shown in green, Fen1 ${ }^{\mathrm{mkikGR}}$ in blue, nCas9 in magenta. Frames collected every minute for $1 \mathrm{~h}$. Corresponding kymograph in Figure 4A,Cul-i.

769

770 Supplemental Video 6. Lag collapse in the presence of p97-i. CMG shown in green, Fen1mkikGR in blue, nCas9 in 771 magenta. Frames collected every minute for $1 \mathrm{~h}$. Corresponding kymograph in Figure 4A,p97-i. 